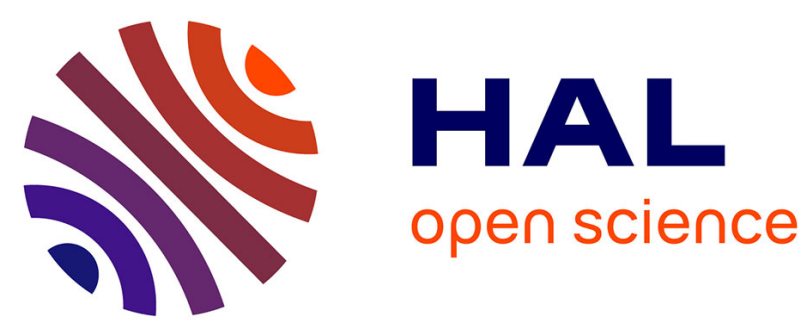

\title{
Application of PGD on parametric modeling of a piezoelectric energy harvester
}

Zhi Qin, Hakeim Talleb, Shuai Yan, Xiaoyu Xu, Zhuoxiang Ren

\section{To cite this version:}

Zhi Qin, Hakeim Talleb, Shuai Yan, Xiaoyu Xu, Zhuoxiang Ren. Application of PGD on parametric modeling of a piezoelectric energy harvester. IEEE Transactions on Magnetics, 2016, 52 (11), pp.7210211. 10.1109/TMAG.2016.2591001 . hal-01358277

\section{HAL Id: hal-01358277 \\ https://hal.sorbonne-universite.fr/hal-01358277}

Submitted on 31 Aug 2016

HAL is a multi-disciplinary open access archive for the deposit and dissemination of scientific research documents, whether they are published or not. The documents may come from teaching and research institutions in France or abroad, or from public or private research centers.
L'archive ouverte pluridisciplinaire HAL, est destinée au dépôt et à la diffusion de documents scientifiques de niveau recherche, publiés ou non, émanant des établissements d'enseignement et de recherche français ou étrangers, des laboratoires publics ou privés. 


\title{
Application of PGD on parametric modeling of a piezoelectric energy harvester
}

\author{
Zhi Qin ${ }^{1}$, Hakeim Talleb ${ }^{1}$, Shuai Yan ${ }^{2}$, Xiaoyu $\mathrm{Xu}^{2}$, and Zhuoxiang Ren ${ }^{1}$ \\ ${ }^{1}$ Sorbonne Universités, Univ. Paris 06, UR2, L2E, F-75005 Paris, France \\ ${ }^{2}$ Institute of Microelectronics of Chinese Academy of Science, Beijing 100029, China
}

In this paper, a priori model reduction methods via low-rank tensor approximation are introduced for the parametric study of a piezoelectric energy harvester (EH). The EH, comprised of a cantilevered piezoelectric bimorph connected with electrical loads, is modeled using three dimensional finite elements (FEs). Solving the model for various excitation frequencies and electrical load using the conventional approach results in a large size problem that is costly in terms of CPU time. We propose an approach based on the proper generalized decomposition (PGD) that can effectively reduce the problem size with a good accuracy of the solutions. With the proposed approach, field variables of the coupled problem are decomposed into space, frequency and electrical load associated components. To introduce PGD into the FE model, a method to model the electrodes and electrical charges in the EH is presented. Appropriate choice for stopping criterions in the method, as well as accelerating the convergence through updating after each enrichment are investigated. The proposed method is validated through a representative numerical example.

Index Terms: Proper generalized decomposition, Model reduction, Finite element method, Piezoelectricity, Energy harvesting.

\section{INTRODUCTION}

$\mathrm{W}$ HEN designing an energy harvester, it is crucial to investigate its performance under various conditions. Take the cantilevered piezoelectric $\mathrm{EH}$ for example, one usually seeks to calculate its output power when the excitation frequency and electrical loads connected between its electrodes vary. The conventional approach is modeling the device with numerical methods such as the FE method while taking the varying variables as parameters. Such parametric modeling leads to high dimensional problems that require a vast amount of computational resource to solve. In practical applications, appropriate methods must be introduced to alleviate the computational complexity.

Recently, researchers have developed various model reduction techniques, in either a posteriori [1] or a priori [2] fashion. The former consists in extracting from available solutions a reduced basis which alleviates the burden in subsequent calculations. Algorithms like the singular value decomposition (SVD), principle component analysis (PCA) and proper orthogonal decomposition (POD) are popular for the purpose. The second category, in contrast, is normally more challenging as it searches a reduced basis without knowing the solution a priori. The proper generalized decomposition is one of the dominant methods under this framework [3]. This work introduces applying the PGD method on the parametric model of a piezoelectric EH.

The essential assumption of the PGD method is that the numerical solution can be expressed as low rank tensors, or a sum of rank-one tensors [4]. Each term in the sum is called a mode while the number of terms is referred to as the tensor rank [5]. From a numerical perspective, each mode is a multi-dimensional array in which every dimension is related to a physical quantity e.g. spatial dimensions and frequency. In practice, modes are calculated successively, which is termed as the enrichment procedure. During each enrichment, simultaneously determining all vectors in the array leads to a nonlinear problem that can be effectively computed using the alternating direction method (ADM) which is sometimes named the fixed point iteration procedure [6]. Advantage of the PGD approach is twofold. Firstly, instead of solving the high-dimensional problem through looping over each sample of the parameter (the conventional approach), it solves the problem with all samples of parameters at the same time, thus avoiding a large number of loops and reducing the CPU time. Secondly, the number of unknowns to be determined is generally dramatically reduced since PGD solutions actually form reduced basis of the conventional solutions. Indeed, for a vast range of problems, only moderate number of enrichments and few iterations within each enrichment are required. Naturally, less solution data needs to be stored which is crucial in real-time simulations [7]. The PGD method also relies on the separability of the problem. Separability of the right hand side (r.h.s.) is usually feasible because excitations and boundary conditions based on which the r.h.s. is formed are normally known. For parameter dependent cases, a posteriori techniques e.g. SVD can be used to obtain a separated representation. On the contrary, separating the coefficient matrix in a general case is still an open problem, albeit some literatures are now available. For instance, in [8] operator separating in both linear and nonlinear cases are discussed. In [9], the same issue in high-dimensional cases are investigated. After separating, PGD formulations can be constructed using the Galerkin orthogonality criteria or residual minimization, depending on whether the operator is self-adjoint [10]. Convergence accelerating methods and optimality of the solution (i.e. attaining the same accuracy with as fewer modes as possible) are also discussed in the same reference. A recently developed approach named ideal minimal residual method is reported in [11] for improving optimality in highdimensional cases. It is based on measuring the residual and the target tensors with appropriately chosen norms. As theoretical framework of the PGD becoming more and more mature, its applications for specific problems are also increasingly visible, such as uncertainty quantification in [12], mechanics in [13], magneto quasi-static problems in [14] and nonlinear magnetic dynamic problems in [15]. 
Although literatures introducing PGD for mechanics or electromagnetics are now available, those involving both mechanics and electromagnetics remain to be exploited. Such problems are particularly important in modeling energy harvesting devices in which energy conversion between mechanical and electromagnetic forms is considered, along with various working conditions and energy storing issues [1617]. It is generally much more computationally expensive to solve these multiphysics problems because the strong coupling effect between physics can significantly increase the problem size, especially in large scale parametric FE models. Here, we propose to apply the PGD method on the FE model of a piezoelectric EH. Although based on appropriate simplifications and developments, analytical models (see e.g. [18]) are less computationally intensive than FE models when dealing with devices of simple geometries and boundary conditions, they become less applicable when the model becomes more sophisticated. FE models, on the other hand, are advantageous due to their versatility in modeling complicated geometry and its capability in obtaining full field solutions under any boundary conditions. In [19], FE methods are utilized for analyzing piezoelectric energy generators using different commercial software modeling multiphysics fields and the electric circuits, individually. Dedicate loops are designed in order to pass results between software. In this paper, PGD is applied so that variables are decomposed into space, frequency and electrical load related components, and the original large-size system is solved through solving a sequence of smaller-size subsystems, thus reducing complexity of the problem. We also introduce a method to incorporate electrodes and electrical loads in the piezoelectric model so that separability of the problem is straightforward which enables getting around separation of the coefficient matrix. In this manner, advantages of the FE model over its analytical counterpart are preserved while the CPU time is significantly decreased. The paper is divided as follows. In section II, formulations of the multiphysics model, with excitation frequency and electrical load as parameters, are derived. In section III, how solution of the model can be approximated with low rank tensors using PGD is introduced. Implementing details are then presented. In Section IV, properties of the proposed method are revealed through a set of numerical illustrations. It finishes with general summaries and conclusions in section $\mathrm{V}$.

\section{FORMULATIONS OF THE MULTIPHYSICS MODEL}

In what follows, quantities of the same uses are expressed in uniform representations. Specifically, lower-case letters are used for scalars; plain capitals for vectors; bold capitals for matrices; and calligraphic letters for tensors of order higher than two.

\section{A. The model problem}

As in most piezoelectric FE models, only linear piezoelectricity [20] is considered here. Assume that we have a threedimensional domain $\Omega_{\varphi} \in \mathbb{R}^{3}$ with Lipschitz boundary $\Gamma_{\varphi}=\Gamma_{\varphi_{\mathrm{D}}} \cup \Gamma_{\varphi_{\mathrm{N}}}$ where $\Gamma_{\varphi_{\mathrm{D}}}$ and $\Gamma_{\varphi_{\mathrm{N}}}$ are the Dirichlet and Neumann electrical boundary conditions. The mechanical domain $\Omega_{\mathrm{u}}$, which is a subdomain of $\Omega_{\varphi}\left(\Omega_{\mathrm{u}} \subset \Omega_{\varphi}\right)$, has its Lipschitz boundary $\Gamma_{\mathrm{u}}=\Gamma_{\mathrm{u}_{\mathrm{D}}} \cup \Gamma_{\mathrm{u}_{\mathrm{N}}}, \Gamma_{\mathrm{u}_{\mathrm{D}}}$ and $\Gamma_{\mathrm{u}_{\mathrm{N}}}$ being, respectively, the Dirichlet and Neumann mechanical boundary condition.

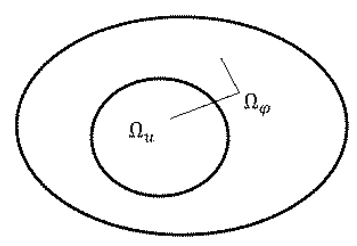

Fig. 1. Electric and mechanical domains of the multiphysics problem

A common practice of modeling piezoelectric materials is to choose $\Omega_{\varphi}$ coinciding with $\Omega_{\mathrm{u}}$ which, however, does not appropriately account for the fringing effect, from an electrical modeling point of view. Here, domains are chosen so that $\Omega_{\varphi} / \Omega_{\mathrm{u}} \neq \varnothing$, as shown in Fig. 1. Indeed, $\Omega_{\varphi} / \Omega_{\mathrm{u}}$ represents an air domain surrounding the piezoelectric part. The strong form of the coupled problem (for detailed derivation refer to [21] and references therein) consists of finding fields of mechanical displacements and electrical potentials subjecting to certain boundary conditions and excitations, as shown in the coupled mechanical equilibrium equation

$$
\begin{aligned}
c_{\mathrm{ijkl}}^{\mathrm{E}} \mathrm{u}_{\mathrm{k}, \mathrm{li}}+\mathrm{e}_{\mathrm{kij}} \varphi_{, \mathrm{ki}}+\mathrm{f}_{\mathrm{j}} & =\rho_{\mathrm{v}} \ddot{\mathrm{u}}_{\mathrm{j}} & & \text { in } \Omega_{\mathrm{u}} \\
\mathrm{u}_{\mathrm{i}} & =\overline{\mathrm{u}} & & \text { on } \Gamma_{\mathrm{u}_{\mathrm{D}}} \\
\mathrm{n}_{\mathrm{i}} \sigma_{\mathrm{ij}} & =\overline{\mathrm{t}} & & \text { on } \Gamma_{\mathrm{u}_{N}}
\end{aligned}
$$

and the coupled electrostatic equation

$$
\begin{aligned}
& \mathrm{e}_{\mathrm{kij}} \mathrm{u}_{\mathrm{i}, \mathrm{jk}}-\varepsilon_{\mathrm{ij}}^{\mathrm{S}} \varphi_{, \mathrm{ij}}=0 \quad \text { in } \Omega_{\mathrm{u}} \\
& -\varepsilon_{\mathrm{ij}}^{\mathrm{S}} \varphi_{, \mathrm{ij}}=0 \quad \text { in } \Omega_{\varphi} / \Omega_{\mathrm{u}} \\
& \varphi=\bar{\varphi} \quad \text { on } \Gamma_{\varphi_{\mathrm{D}}}
\end{aligned}
$$

where the Einsteinian summation convention is employed. $\rho_{\mathrm{v}}$ is the mass density, $\left[\mathrm{c}^{\mathrm{E}}\right],[\mathrm{e}]$, and $\left[\varepsilon^{\mathrm{S}}\right]$ are matrices of elastic, piezoelectric, and dielectric constants, respectively. The superscript $\mathrm{E}$ and $\mathrm{S}$ indicate that values are measured at constant strain and electric field. $\{\mathrm{u}\},\{\mathrm{f}\}$, and $\{\sigma\}$ are respectively, vectors of displacement, body force and stress. $\varphi$ is the electric potential. $\overline{\mathrm{u}}, \overline{\mathrm{t}}$, and $\bar{\varphi}$ are predefined values for respectively, displacements, surface tractions, and electrical potentials. $\mathrm{n}$ denotes the outward normal to the boundary. Repeated indices i, j, k, and l take values1, 2, and 3. A comma subscript followed by an index number means a deriva- 
tion w.r.t. the corresponding direction. Two dots over a variable indicates second order time derivative.

Electrodes are deposited on the piezoelectric part and impose equipotential electrical conditions. Influence of the electrodes are mechanically negligible since they are very thin. Therefore, electrodes can be considered as surfaces and involves merely imposing the equipotential condition. Electrodes and electrical loads are introduced after the fields are approximated using FEs.

\section{B. Finite element approximation}

To approximate the coupled problem using FEs, consider the following weak form. Find $\mathrm{u}_{\mathrm{i}} \in \mathcal{U}$ and $\varphi \in \mathcal{V}$ such that

$$
\begin{array}{cc}
\mathrm{B}_{u u}\left(\mathrm{u}_{\mathrm{j}}^{\prime}, \mathrm{u}_{\mathrm{j}}\right)_{\Omega_{\mathrm{u}}}+\mathrm{B}_{u \varphi}\left(\mathrm{u}_{\mathrm{j}}^{\prime}, \varphi\right)_{\Omega_{\mathrm{u}}}= & \\
-\left(\mathrm{u}_{\mathrm{j}}^{\prime}, \mathrm{f}_{\mathrm{j}}\right)_{\Omega_{\mathrm{u}}}+\left(\mathrm{u}_{\mathrm{j}}^{\prime}, \overline{\mathrm{t}}\right)_{\Gamma_{\mathrm{u}_{\mathrm{N}}}} & \forall \mathrm{u}_{\mathrm{j}}^{\prime} \in \hat{\mathcal{U}} \\
\mathrm{B}_{\varphi \varphi}\left(\varphi^{\prime}, \varphi\right)_{\Omega_{\varphi}}+\mathrm{B}_{\varphi u}\left(\varphi^{\prime}, \mathrm{u}_{\mathrm{i}}\right)_{\Omega_{\mathrm{u}}}=0 & \forall \varphi^{\prime} \in \hat{\mathcal{v}}
\end{array}
$$

where the integrals are defined as

$$
\begin{aligned}
\mathrm{B}_{u u}\left(\mathrm{u}_{\mathrm{j}}^{\prime}, \mathrm{u}_{\mathrm{j}}\right)_{\Omega_{\mathrm{u}}}:=\int_{\Omega_{\mathrm{u}}} \mathrm{u}_{\mathrm{j}}^{\prime}\left(\mathrm{c}_{\mathrm{ijkl}}^{\mathrm{E}} \mathrm{u}_{\mathrm{k}, \mathrm{li}}-\rho_{\mathrm{v}} \ddot{\mathrm{u}}_{\mathrm{j}}\right) \mathrm{d} \Omega \\
\mathrm{B}_{u \varphi}\left(\mathrm{u}_{\mathrm{j}}^{\prime}, \varphi\right)_{\Omega_{\mathrm{u}}}:=\int_{\Omega_{\mathrm{u}}} \mathrm{u}_{\mathrm{j}}^{\prime} \mathrm{e}_{\mathrm{kij}} \varphi_{, \mathrm{ki}} \mathrm{d} \Omega \\
\left(\mathrm{u}_{\mathrm{j}}^{\prime}, \mathrm{f}_{\mathrm{j}}\right)_{\Omega_{\mathrm{u}}}:=\int_{\Omega_{\mathrm{u}}} \mathrm{u}_{\mathrm{j}}^{\prime} \mathrm{f}_{\mathrm{j}} \mathrm{d} \Omega \\
\left(\mathrm{u}_{\mathrm{j}}^{\prime}, \overline{\mathrm{t}}\right)_{\Gamma_{\mathrm{u}_{\mathrm{N}}}}:=\int_{\Gamma_{\mathrm{u}_{\mathrm{N}}}} \mathrm{u}_{\mathrm{j}}^{\prime} \overline{\mathrm{t}} \mathrm{d} \Gamma \\
\mathrm{B}_{\varphi \varphi}\left(\varphi^{\prime}, \varphi\right)_{\Omega_{\varphi}}:=\int_{\Omega_{\varphi}} \varphi^{\prime} \varepsilon_{\mathrm{ij}}^{\mathrm{S}} \varphi_{, \mathrm{ij}} \mathrm{d} \Omega \\
\mathrm{B}_{\varphi u}\left(\varphi^{\prime}, \mathrm{u}_{\mathrm{i}}\right)_{\Omega_{\mathrm{u}}}:=\int_{\Omega_{\mathrm{u}}} \varphi^{\prime} \mathrm{e}_{\mathrm{kij}} \mathrm{u}_{\mathrm{i}, \mathrm{k} \mathrm{k}} \mathrm{d} \Omega
\end{aligned}
$$

The admissible spaces are

$$
\begin{aligned}
& \mathcal{U}:=\left\{\mathrm{u}_{\mathrm{j}} \in \mathrm{H}^{1}\left(\Omega_{\mathrm{u}}\right) \quad \mid \quad \mathrm{u}_{\mathrm{j}}=\overline{\mathrm{u}} \text { on } \Gamma_{\mathrm{u}_{\mathrm{D}}} \mathrm{j}=1,2,3\right\} \\
& \widehat{u}:=\left\{\mathrm{u}_{\mathrm{j}}^{\prime} \in \mathrm{H}^{1}\left(\Omega_{\mathrm{u}}\right) \quad \mid \quad \mathrm{u}_{\mathrm{j}}^{\prime}=0 \text { on } \Gamma_{\mathrm{u}_{\mathrm{D}}}, \mathrm{j}=1,2,3\right\} \\
& \mathcal{V}:=\left\{\varphi \in \mathrm{H}^{1}\left(\Omega_{\varphi}\right) \quad \mid \quad \varphi=\bar{\varphi} \text { on } \Gamma_{\varphi_{\mathrm{D}}}\right\} \\
& \hat{\mathcal{V}}:=\left\{\varphi^{\prime} \in \mathrm{H}^{1}\left(\Omega_{\varphi}\right) \quad \mid \quad \varphi^{\prime}=0 \text { on } \Gamma_{\varphi_{\mathrm{D}}}\right\}
\end{aligned}
$$

where $\mathrm{H}^{1}$ is the first order Sobolev space.

The discrete counterparts of Eq.(3) and Eq.(4) read: find $\mathrm{u}_{\mathrm{jh}} \in \mathcal{U}_{\mathrm{h}}$ and $\varphi_{\mathrm{h}} \in \mathcal{V}_{\mathrm{h}}$ such that

$$
\begin{array}{cc}
\mathrm{B}_{u u}\left(\mathrm{u}_{\mathrm{jh}}{ }^{\prime}, \mathrm{u}_{\mathrm{jh}}\right)_{\Omega_{\mathrm{u}}}+\mathrm{B}_{u \varphi}\left(\mathrm{u}_{\mathrm{jh}}{ }^{\prime}, \varphi_{\mathrm{h}}\right)_{\Omega_{\mathrm{u}}} & \\
=-\left(\mathrm{u}_{\mathrm{jh}}{ }^{\prime}, \mathrm{f}_{\mathrm{j}}\right)_{\Omega_{\mathrm{u}}}+\left(\mathrm{u}_{\mathrm{jh}}{ }^{\prime}, \overline{\mathrm{t}}\right)_{\Gamma_{\mathrm{u}_{\mathrm{N}}}} & \forall \mathrm{u}_{\mathrm{jh}}{ }^{\prime} \in \hat{\mathcal{U}}_{\mathrm{h}} \\
\mathrm{B}_{\varphi \varphi}\left(\varphi_{\mathrm{h}}{ }^{\prime}, \varphi_{\mathrm{h}}\right)_{\Omega_{\varphi}}+\mathrm{B}_{\varphi u}\left(\varphi_{\mathrm{h}}{ }^{\prime}, \mathrm{u}_{\mathrm{ih}}{ }^{\prime}\right)_{\Omega_{\mathrm{u}}}=0 \quad \forall \varphi_{\mathrm{h}} \in \hat{\mathcal{V}}_{\mathrm{h}}
\end{array}
$$

where $\mathcal{U}_{\mathrm{h}} \subset \mathcal{U}, \hat{U}_{\mathrm{h}} \subset \hat{u}, \mathcal{v}_{\mathrm{h}} \subset \mathcal{v}$, and $\hat{v}_{\mathrm{h}} \subset \hat{v}$ are finite dimensional spaces, with $\mathrm{h}$ characterizing the triangulation resolution. Within a given finite element $e$ of domain $\Omega_{\mathrm{u}}^{\mathrm{e}}$ (resp. $\Omega_{\varphi}^{\mathrm{e}}$ ), the discretized mechanical (resp. electrical) test and trial functions can be constructed using the Galerkin method, as in Eq.(9) .

$$
\begin{aligned}
u_{j h}{ }^{\prime} & =\sum_{m=1}^{n_{u}} N_{m}^{(u)} u_{j_{m}}^{e} \\
u_{j h} & =\sum_{m=1}^{n_{u}} N_{m}^{(u)} u_{j_{m}}^{e}
\end{aligned}
$$

and Eq.(10)

$$
\begin{aligned}
\varphi_{\mathrm{h}}{ }^{\prime} & =\sum_{\mathrm{m}=1}^{\mathrm{n}_{\varphi}} \mathrm{N}_{\mathrm{m}}^{(\varphi)} \varphi_{\mathrm{m}}^{\mathrm{e}} \\
\varphi_{\mathrm{h}} & =\sum_{\mathrm{m}=1}^{\mathrm{n}_{\varphi}} \mathrm{N}_{\mathrm{m}}^{(\varphi)} \varphi_{\mathrm{m}}^{\mathrm{e}}
\end{aligned}
$$

where $\mathrm{n}_{\mathrm{u}}$ and $\mathrm{n}_{\varphi}$ are total number of nodes in the mechanical and electrical domain respectively. $\mathrm{N}_{\mathrm{m}}^{(\mathrm{u})}$ and $\mathrm{N}_{\mathrm{m}}^{(\varphi)}$ are shape functions and the same shape function $\mathrm{N}_{\mathrm{m}}^{(\mathrm{u})}$ is used for all components. $\mathrm{u}_{\mathrm{j}_{\mathrm{m}}}^{\prime \mathrm{e}}, \mathrm{u}_{\mathrm{j}_{\mathrm{m}}}^{\mathrm{e}}, \varphi_{\mathrm{m}}^{\prime \mathrm{e}}$ and $\varphi_{\mathrm{m}}^{\mathrm{e}}$ are nodal values.

In the implementation, it is more convenient to arrange $\mathrm{u}_{\mathrm{jh}}{ }^{\prime} \mathrm{s}$ and $\varphi_{\mathrm{h}}{ }^{\prime} \mathrm{s}$ in vectors. For instance, $\mathrm{u}_{\mathrm{jh}}{ }^{\prime} \mathrm{s}$ can be written as $\mathrm{U}_{\mathrm{h}}=\left[\begin{array}{lll}\mathrm{u}_{1 \mathrm{~h}} & \mathrm{u}_{2 \mathrm{~h}} & \mathrm{u}_{3 \mathrm{~h}}\end{array}\right]^{\mathrm{T}}$. Re-writing the above equations in the compact form yields

$$
\begin{gathered}
\mathrm{U}_{\mathrm{h}}{ }^{\prime}=\mathrm{N}_{\mathrm{u}}^{\mathrm{e}} \mathrm{U}^{\prime}, \quad \mathrm{U}_{\mathrm{h}}=\mathrm{N}_{\mathrm{u}}^{\mathrm{e}} \mathrm{U} \\
\varphi_{\mathrm{h}}{ }^{\prime}=\mathrm{N}_{\varphi}^{\mathrm{e}} \Phi^{\prime}, \quad \varphi_{\mathrm{h}}=\mathrm{N}_{\varphi}^{\mathrm{e}} \Phi
\end{gathered}
$$

where

$$
\begin{aligned}
\mathrm{U}^{\prime} & =\left[\begin{array}{lllll}
\mathrm{u}_{11}^{\prime} & \mathrm{u}_{21}^{\prime} & \mathrm{u}_{31}^{\prime}{ }_{31}^{\mathrm{e}} & \cdots & \mathrm{u}_{3_{\mathrm{n}_{\mathrm{u}}}^{\prime}}^{\mathrm{e}}
\end{array}\right]^{T} \\
\mathrm{U} & =\left[\begin{array}{lllll}
\mathrm{u}_{1} \mathrm{e} & \mathrm{u}_{21}^{\mathrm{e}} & \mathrm{u}_{31}^{\mathrm{e}} & \cdots & \mathrm{u}_{3} \mathrm{n}_{\mathrm{u}}
\end{array}\right]^{T} \\
\Phi^{\prime} & =\left[\begin{array}{llll}
\varphi_{1}^{\prime} & \varphi_{2}^{\prime} & \cdots & \varphi_{\mathrm{n}_{\varphi}}^{\mathrm{e}}
\end{array}\right]^{\mathrm{T}} \\
\Phi & =\left[\begin{array}{llll}
\varphi_{1}^{\mathrm{e}} & \varphi_{2}^{\mathrm{e}} & \cdots & \varphi_{\mathrm{n}_{\varphi}}^{\mathrm{e}}
\end{array}\right]^{\mathrm{T}} \\
\mathbf{N}_{\mathrm{u}}^{\mathrm{e}} & =\left[\begin{array}{lllll}
\mathrm{N}_{1}^{(\mathrm{u})} \mathbf{I}_{3} & \mathrm{~N}_{2}^{(\mathrm{u})} \mathbf{I}_{3} & \cdots & \mathrm{N}_{\mathrm{n}_{\mathrm{u}}}^{(\mathrm{u})} \mathbf{I}_{3}
\end{array}\right] \\
\mathrm{N}_{\varphi}^{\mathrm{e}} & =\left[\begin{array}{llll}
\mathrm{N}_{1}^{(\varphi)} & \mathrm{N}_{2}^{(\varphi)} & \cdots & \mathrm{N}_{\mathrm{n}_{\varphi}}^{(\varphi)}
\end{array}\right]
\end{aligned}
$$

and $\mathbf{I}_{3}$ is a $3 \times 3$ identity matrix.

The FE system can be obtained through substituting Eq.(11) into Eq.(7) and Eq.(8) and integrating. Matrices and vectors are then calculated element-wise: for the $\alpha_{\text {th }}$ element $\mathrm{e}_{\alpha} \in \Omega_{\mathrm{u}}$

$$
\begin{aligned}
& \mathbf{M}_{\mathrm{u}}^{(\alpha)} \ddot{\mathrm{U}}_{\alpha}+\mathbf{K}_{\mathrm{u}}^{(\alpha)} \mathrm{U}_{\alpha}+\mathbf{K}_{\mathrm{u} \varphi}^{(\alpha)} \Phi_{\alpha}=\mathrm{F}_{\mathrm{X}}^{(\alpha)} \\
& \mathbf{K}_{\mathrm{u} \varphi}^{(\alpha), \mathrm{T}} \mathrm{U}_{\alpha}-\mathbf{K}_{\varphi}^{(\alpha)} \Phi_{\alpha}=0
\end{aligned}
$$

and for the $\beta_{\text {th }}$ element $\mathrm{e}_{\beta} \in \Omega_{\varphi} / \Omega_{\mathrm{u}}$

$$
-\mathbf{K}_{\varphi}^{(\beta)} \Phi_{\beta}=0
$$


where $\mathrm{U}_{\alpha}$ and $\Phi_{\alpha}$ (resp. $\Phi_{\beta}$ ) represent all nodal values within the element $\mathrm{e}_{\alpha}$ (resp. $\mathrm{e}_{\beta}$ ). Without losing generality (w.l.g.), assume that linear tetrahedral elements are used. Then, the vector $\Phi_{\alpha}$ equal to $\left[\varphi_{\alpha 1}^{\mathrm{e}} \quad \varphi_{\alpha 2}^{\mathrm{e}} \quad \varphi_{\alpha 3}^{\mathrm{e}} \quad \varphi_{\alpha 4}^{\mathrm{e}}\right]$ is of size $4 \times 1$ and $U_{\alpha}$ is of size $12 \times 1, \alpha 1$ to $\alpha 4$ being node numerations in element $\alpha$. The mass matrix $\mathbf{M}_{\mathrm{u}}^{(\alpha)}$, stiffness matrices $\mathbf{K}_{\mathrm{u}}^{(\alpha)}$ and $\mathbf{K}_{\varphi}^{(\alpha)}$, coupling matrix $\mathbf{K}_{\mathrm{u} \varphi}^{(\alpha)}$ and the mechanical load vector $\mathrm{F}_{\mathrm{X}}{ }^{(\alpha)}$ are calculated as follows.

$$
\begin{aligned}
\mathbf{M}_{\mathrm{u}}^{(\alpha)} & =\int_{\Omega_{\mathrm{e}_{\alpha}}} \mathbf{N}_{\mathrm{u}}^{\mathrm{e}_{\alpha}, \mathrm{T}} \rho \mathbf{N}_{\mathrm{u}}^{\mathrm{e}_{\alpha}} \mathrm{d} \Omega \\
\mathbf{K}_{\mathrm{u}}^{(\alpha)} & =\int_{\Omega_{\mathrm{e}_{\alpha}}} \mathbf{B}_{\mathrm{u}}^{\mathrm{e}_{\alpha}, \mathrm{T}}\left[\mathrm{c}^{\mathrm{E}}\right] \mathbf{B}_{\mathrm{u}}^{\mathrm{e}_{\alpha}} \mathrm{d} \Omega \\
\mathbf{K}_{\mathrm{u} \varphi}^{(\alpha)} & =\int_{\Omega_{\mathrm{e}_{\alpha}}} \mathbf{B}_{\mathrm{u}}^{\mathrm{e}_{\alpha}, \mathrm{T}}[\mathrm{e}]^{T} \mathbf{B}_{\varphi}^{\mathrm{e}_{\alpha}} \mathrm{d} \Omega \\
\mathbf{K}_{\varphi}^{(\alpha, \beta)} & =\int_{\Omega_{\mathrm{e}_{\alpha, \beta}}} \mathbf{B}_{\varphi}^{\mathrm{e}_{\alpha,}, \mathrm{T}}\left[\varepsilon^{\mathrm{S}}\right] \mathbf{B}_{\varphi}^{\mathrm{e}_{\alpha}} \mathrm{d} \Omega \\
\mathrm{F}_{\mathrm{X}}{ }^{(\alpha)} & =\int_{\Omega_{\mathrm{e}_{\alpha}}} \mathbf{N}_{\mathrm{u}}^{\mathrm{e}_{\alpha, \mathrm{T}}} \rho\{\mathrm{f}\} \mathrm{d} \Omega+\int_{\Gamma_{\mathrm{u}_{\mathrm{N}}} \cap \partial \Omega_{\mathrm{e}_{\alpha}}} \mathbf{N}_{\mathrm{u}}^{\mathrm{e}_{\alpha}, \mathrm{T}} \rho \overline{\mathrm{t}} \mathrm{d} \Gamma
\end{aligned}
$$

in which the matrices are defined as

$$
\begin{aligned}
& \mathbf{B}_{\mathrm{u}}^{\mathrm{e}_{\alpha}}=\left[\begin{array}{llll}
\mathbf{B}_{\mathrm{u}}^{\mathrm{e}_{\alpha 1}} & \mathbf{B}_{\mathrm{u}}^{\mathrm{e}_{\alpha 2}} & \mathbf{B}_{\mathrm{u}}^{\mathrm{e}_{\alpha 3}} & \mathbf{B}_{\mathrm{u}}^{\mathrm{e}_{\alpha 4}}
\end{array}\right] \\
& \mathbf{B}_{\varphi}^{\mathbf{e}_{\alpha}}=\left[\begin{array}{cccc}
\frac{\partial \mathrm{N}_{\alpha 1}^{(\varphi)}}{\partial \mathrm{x}_{1}} & \frac{\partial \mathrm{N}_{\alpha 2}^{(\varphi)}}{\partial \mathrm{x}_{1}} & \frac{\partial \mathrm{N}_{\alpha 3}^{(\varphi)}}{\partial \mathrm{x}_{1}} & \frac{\partial \mathrm{N}_{\alpha 4}^{(\varphi)}}{\partial \mathrm{x}_{1}} \\
\frac{\partial \mathrm{N}_{\alpha 1}^{(\varphi)}}{\partial \mathrm{x}_{2}} & \frac{\partial \mathrm{N}_{\alpha 2}^{(\varphi)}}{\partial \mathrm{x}_{2}} & \frac{\partial \mathrm{N}_{\alpha 3}^{(\varphi)}}{\partial \mathrm{x}_{2}} & \frac{\partial \mathrm{N}_{\alpha 4}^{(\varphi)}}{\partial \mathrm{x}_{2}} \\
\frac{\partial \mathrm{N}_{\alpha 1}^{(\varphi)}}{\partial \mathrm{x}_{3}} & \frac{\partial \mathrm{N}_{\alpha 2}^{(\varphi)}}{\partial \mathrm{x}_{3}} & \frac{\partial \mathrm{N}_{\alpha 3}^{(\varphi)}}{\partial \mathrm{x}_{3}} & \frac{\partial \mathrm{N}_{\alpha 4}^{(\varphi)}}{\partial \mathrm{x}_{3}}
\end{array}\right] \\
& \mathbf{N}_{\mathrm{u}}^{\mathrm{e}_{\alpha}}=\left[\begin{array}{llll}
\mathrm{N}_{\alpha 1}^{(\mathrm{u})} \mathbf{I}_{3} & \mathrm{~N}_{\alpha 2}^{(\mathrm{u})} \mathbf{I}_{3} & \mathrm{~N}_{\alpha 3}^{(\mathrm{u})} \mathbf{I}_{3} & \mathrm{~N}_{\alpha 4}^{(\mathrm{u})} \mathbf{I}_{3}
\end{array}\right] \\
& \mathbf{B}_{\mathrm{u}}^{\mathrm{e}_{\alpha \mathrm{i}}}=\left[\begin{array}{cccccc}
\frac{\partial \mathrm{N}_{\alpha \mathrm{i}}^{(\mathrm{u})}}{\partial \mathrm{x}_{1}} & 0 & 0 & \frac{\partial \mathrm{N}_{\alpha \mathrm{i}}^{(\mathrm{u})}}{\partial \mathrm{x}_{2}} & 0 & \frac{\partial \mathrm{N}_{\alpha \mathrm{i}}^{(\mathrm{u})}}{\partial \mathrm{x}_{3}} \\
0 & \frac{\partial \mathrm{N}_{\alpha \mathrm{i}}^{(\mathrm{u})}}{\partial \mathrm{x}_{2}} & 0 & \frac{\partial \mathrm{N}_{\alpha \mathrm{i}}^{(\mathrm{u})}}{\partial \mathrm{x}_{1}} & \frac{\partial \mathrm{N}_{\alpha \mathrm{i}}^{(\mathrm{u})}}{\partial \mathrm{x}_{3}} & 0 \\
0 & 0 & \frac{\partial \mathrm{N}_{\alpha \mathrm{i}}^{(\mathrm{u})}}{\partial \mathrm{x}_{3}} & 0 & \frac{\partial \mathrm{N}_{\alpha \mathrm{i}}^{(\mathrm{u})}}{\partial \mathrm{x}_{2}} & \frac{\partial \mathrm{N}_{\alpha \mathrm{i}}^{(\mathrm{u})}}{\partial \mathrm{x}_{1}}
\end{array}\right]^{T}
\end{aligned}
$$

To model the structural damping, Rayleigh damping can be added which introduces the damping matrix as a fraction of the mass and stiffness matrix using $\alpha_{\mathrm{dM}}$ and $\beta_{\mathrm{dK}}$.

$$
\mathbf{C}_{\mathrm{u}}^{(\alpha)}=\alpha_{\mathrm{dM}} \mathbf{M}_{\mathrm{u}}^{(\alpha)}+\beta_{\mathrm{dK}} \mathbf{K}_{\mathrm{u}}^{(\alpha)}
$$

The parameters $\alpha_{\mathrm{dM}}$ and $\beta_{\mathrm{dK}}$ can be obtained from Eq.(17) where $\xi$ denotes the damping factor at resonant frequency $\omega_{0}$. Damping factors can be measured at the first two resonant frequencies with which Rayleigh damping are calculated and used for the whole frequency range, approximately [18].

$$
\xi=\frac{1}{2}\left(\frac{\alpha_{\mathrm{dM}}}{\omega_{0}}+\beta_{\mathrm{dK}} \omega_{0}\right)
$$

Adding Eq.(16) into Eq.(13.a) leads to

$$
\mathbf{M}_{\mathrm{u}}^{(\alpha)} \ddot{\mathrm{U}}_{\alpha}^{\mathrm{e}}+\mathbf{C}_{\mathrm{u}}^{(\alpha)} \dot{\mathrm{U}}_{\alpha}^{\mathrm{e}}+\mathbf{K}_{\mathrm{u}}^{(\alpha)} \mathrm{U}_{\alpha}^{\mathrm{e}}+\mathbf{K}_{\mathrm{u} \varphi}^{(\alpha)} \Phi_{\alpha}^{\mathrm{e}}=\mathrm{F}_{\mathrm{X}}{ }^{(\alpha)}
$$

Numerically, damping also prevents singularity when Eq.(18) is solved near frequencies of resonance in the frequency domain. Before assembling elementary matrices into a global system that applies to the whole domain, modeling of the electrodes is addressed.

\section{Modeling the electrodes and electrical loads}

Denote the surface of the $\mathrm{k}_{\mathrm{th}}$ electrode as $\mathrm{S}_{\mathrm{e}}^{\mathrm{k}}$. The equipotential condition imposed by the electrode means $\varphi(X)=$ $\varphi_{\text {const }}$ for $\mathrm{X} \in \mathrm{S}_{\mathrm{e}}^{\mathrm{k}}$. When the electric potential is prescribed, it can be fulfilled by imposing Dirichlet boundary conditions. However, when $\varphi_{\text {const }}$ is not prescribed it needs to be treated with caution. Suppose there are $n_{\mathrm{e}}^{\mathrm{k}}$ mesh nodes on $\mathrm{S}_{\mathrm{e}}^{\mathrm{k}}$. The number of electrical degree of freedoms (dofs) is reduced to one for the $\mathrm{n}_{\mathrm{e}}^{\mathrm{k}}$ nodes. Denote the mapping that defines numeration of the electrical dof for each node as $\mathcal{M}_{\mathrm{dof}}^{\varphi}$.W.1.g., assume that all $\mathrm{n}_{\mathrm{e}}^{\mathrm{k}}$ electrical dofs are assigned with the number $\mathrm{k}\left(\mathcal{M}_{\mathrm{dof}}^{\varphi}\left(\mathrm{X} \in \mathrm{S}_{\mathrm{e}}^{\mathrm{k}}\right)=\mathrm{k}\right)$. If there are $\mathrm{m}_{\mathrm{e}}$ electrodes, then the total number of electrical dofs that are not on electrodes is $\mathrm{n}_{\varphi}-\sum_{\mathrm{k}=1}^{\mathrm{m}_{\mathrm{e}}} \mathrm{n}_{\mathrm{e}}^{\mathrm{k}}$. These dofs can be assigned with number from $\mathrm{m}_{\mathrm{e}}+1$ to $\mathrm{m}_{\mathrm{e}}+\mathrm{n}_{\varphi}-\sum_{\mathrm{k}=1}^{\mathrm{m}_{\mathrm{e}}} \mathrm{n}_{\mathrm{e}}^{\mathrm{k}}-\mathrm{n}_{\widehat{\varphi}}, \mathrm{n}_{\widehat{\varphi}}$ denoting the number of nodes on $\Gamma_{\varphi_{\mathrm{D}}}$. Meanwhile, the mechanical mapping $\mathcal{M}_{\text {dof }}^{\mathrm{u}}$ can be such defined that the $\mathrm{j}_{\text {th }}$ component of the dofs at the $l_{\text {th }}$ mesh node is assigned $3 \times \mathrm{l}+\mathrm{j}-1$ with $j=1,2,3$. Hence, the total number of mechanical and electrical dofs $\mathrm{N}_{\mathrm{u}}$ and $\mathrm{N}_{\varphi}$ are respectively, $3 \mathrm{n}_{\mathrm{u}}-\mathrm{n}_{\widehat{\mathrm{u}}}$ and $\mathrm{m}_{\mathrm{e}}+$ $\mathrm{n}_{\varphi}-\mathrm{n}_{\widehat{\varphi}}-\sum_{\mathrm{k}=1}^{\mathrm{m}_{\mathrm{e}}} \mathrm{n}_{\mathrm{e}}^{\mathrm{k}}, \mathrm{n}_{\widehat{\mathrm{u}}}$ being the number of nodes on $\Gamma_{\mathrm{u}_{\mathrm{D}}}$. In assembling, positions of each elementary matrix and vector in the global system are determined by the mapping results. The global system can be written as

$$
\begin{aligned}
\mathbf{M}_{\mathrm{u}} \ddot{\mathrm{U}}+\mathbf{C}_{\mathrm{u}} \dot{U}+\mathbf{K}_{\mathrm{u}} \mathrm{U}+\mathbf{K}_{\mathrm{u} \varphi} \Phi & =\mathrm{F}_{\mathrm{X}} \\
\mathbf{K}_{\mathrm{u} \varphi}^{\mathrm{T}} \mathrm{U}-\mathbf{K}_{\varphi} \Phi & =0
\end{aligned}
$$

where $\mathbf{M}_{\mathrm{u}} \in \mathbb{R}^{\mathrm{N}_{\mathrm{u}} \times \mathrm{N}_{\mathrm{u}}}, \mathbf{C}_{\mathrm{u}} \in \mathbb{R}^{\mathrm{N}_{\mathrm{u}} \times \mathrm{N}_{\mathrm{u}}}, \mathbf{K}_{\mathrm{u}} \in \mathbb{R}^{\mathrm{N}_{\mathrm{u}} \times \mathrm{N}_{\mathrm{u}}}, \mathbf{K}_{\mathrm{u} \varphi} \in$ $\mathbb{R}^{\mathrm{N}_{\mathrm{u}} \times \mathrm{N}_{\varphi}}, \mathbf{K}_{\varphi} \in \mathbb{R}^{\mathrm{N}_{\varphi} \times \mathrm{N}_{\varphi}}$, and $\mathrm{F}_{\mathrm{X}} \in \mathbb{R}^{\mathrm{N}_{\mathrm{u}} \times 1}$.

Eq.(19) can be transformed from the time domain to the frequency domain. For a time-harmonic excitation at angular frequency $\omega$, the transforming yields

$$
\begin{aligned}
\left(-\omega^{2} \mathbf{M}_{\mathrm{u}}+\mathrm{j} \omega \mathbf{C}_{\mathrm{u}}+\mathbf{K}_{\mathrm{u}}\right) \mathrm{U}+\mathbf{K}_{\mathrm{u} \varphi} \Phi & =\mathrm{F}_{\mathrm{X}} \\
\mathbf{K}_{\mathrm{u} \varphi}^{\mathrm{T}} \mathrm{U}-\mathbf{K}_{\varphi} \Phi & =0
\end{aligned}
$$

where $\mathrm{j}$ is the imaginary unit.

Incorporating electric circuits into Eq.(20) can be achieved via describing each circuit element with the so called equivalent capacitance matrix [22] and adding the resultant matrices into the dielectric matrix of Eq.(20.b). To be more precise, $\mathbf{K}_{\varphi}$ is replaced with $\mathbf{K}_{\varphi}+\widehat{\mathbf{K}}_{\varphi}$ in which the effective 
capacitance matrix $\widehat{\mathbf{K}}_{\varphi}$ is initially null. For each circuit element $\check{x}$ connected between the $p_{\text {th }}$ and $q_{\text {th }}$ electrode, define a vector $\mathrm{V}_{\breve{\mathrm{x}}}$ that is of size $\mathrm{N}_{\varphi} \times 1$ and its $\mathrm{p}_{\text {th }}$ and $\mathrm{q}_{\text {th }}$ component are respectively 1 and -1 while others are zero.

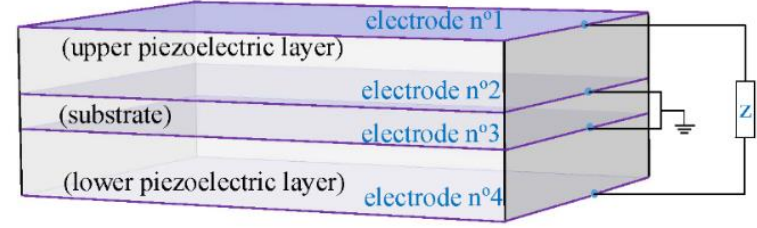

Fig. 2. Piezoelectric EH connected to electrical loads in series

Denote $\breve{\mathbf{K}}_{\varphi \breve{\mathrm{x}}}$ as the product $\mathrm{V}_{\breve{\mathrm{X}}} \mathrm{V}_{\breve{\mathrm{x}}}^{\mathrm{T}}$ (the incident matrix) for conciseness. Then the matrix $-\mathrm{j} / \omega \widetilde{Z}_{\breve{\mathbf{x}}} \breve{\mathbf{K}}_{\varphi \check{\mathrm{x}}}$ is added to $\widehat{\mathbf{K}}_{\varphi}$.

$$
\widehat{\mathbf{K}}_{\varphi}=\sum_{\check{\mathrm{x}}} \frac{\mathrm{j}}{\omega \widetilde{Z}_{\check{x}}} \breve{\mathbf{K}}_{\varphi \check{x}}
$$

$\tilde{Z}_{\breve{X}}$ equals to $-Z_{\breve{X}}$ when $\check{\mathrm{X}}$ is a capacitor; while $\tilde{Z}_{\breve{\mathrm{X}}}=\mathrm{Z}_{\breve{\mathrm{X}}}$ when $\check{X}$ is a resistor or an inductor, with $Z_{\breve{X}}$ denoting the impedance of $\breve{x}$. The new global system can be expressed as

$$
\begin{array}{r}
\left(-\omega^{2} \mathbf{M}_{\mathrm{u}}+\mathrm{j} \omega \mathbf{C}_{\mathrm{u}}+\mathbf{K}_{\mathrm{u}}\right) \mathrm{U}+\mathbf{K}_{\mathrm{u} \varphi} \Phi=\mathrm{F}_{\mathrm{X}} \\
\mathbf{K}_{\mathrm{u} \varphi}^{\mathrm{T}} \mathrm{U}-\left(\mathbf{K}_{\varphi}+\widehat{\mathbf{K}}_{\varphi}\right) \Phi=0
\end{array}
$$

For EH design, one of the most frequently used configuration is that composed of a piezoelectric bimorph connected with a resistor in series, as represented in Fig.2. In this case, the electrodes 2 and 3 are grounded while the resistor is connected between electrodes 1 and 4 . Consequently, the effective capacitance matrix can be simplified as $-\mathrm{j} / \omega \mathrm{r} \breve{\mathbf{K}}_{\varphi}$. This configuration is condidered in the following.

The above equation is multi-parametric, depending on $\omega$ and r. Usually, results of the FE model for a wide range of these parameters are of interest. For instance, one may seek the EH output for all combinations of $\omega$ in the $\mathrm{N}_{\omega} \times 1$ vector $\mathrm{V}_{\omega}:=\left[\begin{array}{llll}\omega_{1} & \omega_{2} & \cdots & \omega_{\mathrm{N}_{\omega}}\end{array}\right]$ and $\mathrm{r}$ in the $\mathrm{N}_{\mathrm{r}} \times 1$ vector $V_{r}:=\left[\begin{array}{llll}r_{1} & r_{2} & \cdots & r_{N_{r}}\end{array}\right]$, so as to find the optimal working condition. A conventional approach to solve the problems is to solve Eq.(22) with two loops for $\omega$ and $r$, one comprised in the other. This approach leads to the model complexity increasing exponentially with the number of parameters. This approach takes a huge amount of time, especially when the total number of parameters is large.

\section{PGD BASED MODEL ORDER REDUCTION}

In this section, a novel approach that enables the complexity increase linearly with the number of parameters is introduced. Note that when Eq.(22) is solved for values of $\omega$ and $r$, solutions can be arranged as three-way arrays $\mathfrak{U}$ and $\mathfrak{B}$, respectively for the displacements and potentials. Each dimension in the array relates to either the spatial coordinates, frequencies or resistors. The goal with the novel ap- proach is to approximate $\mathfrak{U}$ and $\mathfrak{B}$ in terms of low rank tensors in the canonical form [23], as depicted in Fig.3 where $\mathfrak{Y}$ stands for $\mathfrak{U}$ or $\mathfrak{B}$.

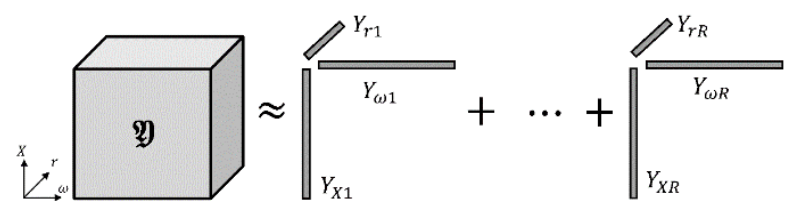

Fig. 3. Approximation of the solution via low rank tensors

\section{A. Separated representations}

Expressing the above figure in equations yields

$$
\begin{aligned}
& \mathfrak{U}(\mathrm{X}, \omega, \mathrm{r}) \approx \sum_{\mathrm{i}=1}^{\mathrm{R}} \mathrm{U}_{\mathrm{Xi}} \otimes \mathrm{U}_{\omega \mathrm{i}} \otimes \mathrm{U}_{\mathrm{ri}} \\
& \mathfrak{V}(\mathrm{X}, \omega, \mathrm{r}) \approx \sum_{\mathrm{i}=1}^{\mathrm{R}} \Phi_{\mathrm{Xi}} \otimes \Phi_{\omega \mathrm{i}} \otimes \Phi_{\mathrm{ri}}
\end{aligned}
$$

where $\mathrm{U}_{\mathrm{Xi}} \in \mathbb{C}^{\mathrm{N}_{\mathrm{u}} \times 1}, \Phi_{\mathrm{Xi}} \in \mathbb{C}^{\mathrm{N}_{\varphi} \times 1}, \mathrm{U}_{\omega \mathrm{i}}, \Phi_{\omega \mathrm{i}} \in \mathbb{C}^{\mathrm{N}_{\omega} \times 1}$, and $\mathrm{U}_{\mathrm{ri}}, \Phi_{\mathrm{ri}} \in \mathbb{C}^{\mathrm{N}_{\mathrm{r}} \times 1}$. ' $\otimes$ ' denotes the Kronecker product. $\mathrm{R}$ is the total number of modes.

Note that decompositions in Eq.(23) is the solution of a system obtained by expanding Eq.(22) for all samples of the parameters. To obtain this system, Eq.(22) needs to be expanded in tensor form. For the r.h.s., w.l.g., assume that identical mechanical excitations are applied in all cases of $\omega$ and r. Thus, r.h.s. in Eq.(22.a) is expanded as $F_{X} \otimes F_{\omega} \otimes F_{r}$ where $F_{\omega}$ and $F_{r}$, whose components are all equal to 1 , are vectors of size $\mathrm{N}_{\omega} \times 1$ and $\mathrm{N}_{\mathrm{r}} \times 1$, respectively. Expanding matrices in Eq.(22) in tensor form is straightforward as variables related to $\mathrm{X}, \omega$ and $\mathrm{r}$ are individual terms in products, thanks to the method modeling electrodes and electrical loads. The resulting tensor comprises four blocks

$$
\mathfrak{U}=\left[\begin{array}{ll}
\mathfrak{U}_{11} & \mathfrak{A}_{12} \\
\mathfrak{A}_{21} & \mathfrak{A}_{22}
\end{array}\right]
$$

with

$$
\begin{aligned}
\mathfrak{U}_{11}= & -\mathbf{M}_{\mathrm{u}} \otimes \mathbf{A}_{\omega} \otimes \mathbf{D}_{\mathrm{r}}+\mathrm{j} \mathbf{C}_{\mathrm{u}} \otimes \mathbf{B}_{\omega} \otimes \mathbf{D}_{\mathrm{r}} \\
& +\mathbf{K}_{\mathrm{u}} \otimes \mathbf{D}_{\omega} \otimes \mathbf{D}_{\mathrm{r}} \\
\mathfrak{A}_{12}= & \mathbf{K}_{\mathrm{u} \varphi} \otimes \mathbf{D}_{\omega} \otimes \mathbf{D}_{\mathrm{r}} \\
\mathfrak{A}_{21}= & \mathbf{K}_{\mathrm{u} \varphi}^{\mathrm{T}} \otimes \mathbf{D}_{\omega} \otimes \mathbf{D}_{\mathrm{r}} \\
\mathfrak{A}_{22}= & \mathbf{K}_{\varphi} \otimes \mathbf{D}_{\omega} \otimes \mathbf{D}_{\mathrm{r}}-j \breve{\mathbf{K}}_{\varphi} \otimes \mathbf{G}_{\omega} \otimes \mathbf{G}_{\mathrm{r}}
\end{aligned}
$$

where $\mathbf{D}_{\omega}$ (resp. $\mathbf{D}_{\mathrm{r}}$ ) equals to $\mathbf{I}_{\mathrm{N}_{\omega}}$ (resp. $\mathbf{I}_{\mathrm{N}_{\mathrm{r}}}$ ), the identity $\mathrm{N}_{\omega} \times \mathrm{N}_{\omega}\left(\right.$ resp. $\left.\mathrm{N}_{\mathrm{r}} \times \mathrm{N}_{\mathrm{r}}\right)$ matrix. The others are defined as

$$
\begin{aligned}
& \mathbf{A}_{\omega}:=\operatorname{diag}\left\{\omega_{1}^{2} \quad \omega_{2}^{2} \quad \cdots \quad \omega_{\mathrm{N}_{\omega}}^{2}\right\} \in \mathbb{R}^{\mathrm{N}_{\omega} \times \mathrm{N}_{\omega}} \\
& \mathbf{B}_{\omega}:=\operatorname{diag}\left\{\omega_{1} \quad \omega_{2} \quad \cdots \quad \omega_{N_{\omega}}\right\} \in \mathbb{R}^{N_{\omega} \times N_{\omega}} \\
& \mathbf{G}_{\omega}:=\operatorname{diag}\left\{1 / \omega_{1} \quad 1 / \omega_{2} \quad \cdots \quad 1 / \omega_{\mathrm{N}_{\omega}}\right\} \in \mathbb{R}^{\mathrm{N}_{\omega} \times \mathrm{N}_{\omega}} \\
& \mathbf{G}_{\mathrm{r}}:=\operatorname{diag}\left\{\begin{array}{llll}
1 / \mathrm{r}_{1} & 1 / \mathrm{r}_{2} & \cdots & 1 / \mathrm{r}_{\mathrm{N}_{\mathrm{r}}}
\end{array}\right\} \in \mathbb{R}^{\mathrm{N}_{\mathrm{r}} \times \mathrm{N}_{\mathrm{r}}}
\end{aligned}
$$


To keep notations neat, modes in Eq.(23) are designated as

$$
\mathfrak{x}^{\mathrm{i}}:=\left[\mathfrak{x}_{1}^{\mathrm{i}}, \mathfrak{x}_{2}^{\mathrm{i}}\right]^{\mathrm{T}}=\left[\mathrm{U}_{\mathrm{Xi}} \otimes \mathrm{U}_{\omega \mathrm{i}} \otimes \mathrm{U}_{\mathrm{ri}}, \Phi_{\mathrm{Xi}} \otimes \Phi_{\omega \mathrm{i}} \otimes \Phi_{\mathrm{ri}}\right]^{\mathrm{T}}
$$

and the expanded r.h.s.s as

$$
\mathfrak{b}:=\left[\mathfrak{b}_{1}, \mathfrak{b}_{2}\right]^{\mathrm{T}}=\left[\mathrm{F}_{\mathrm{X}} \otimes \mathrm{F}_{\omega} \otimes \mathrm{F}_{\mathrm{r}}, 0\right]^{\mathrm{T}}
$$

Subscript 1 and 2 indicate association with mechanical and electrical variables, respectively. Components associated with $X, \omega$ and $r$ are accessed with an additional subscript $\kappa$ with $\kappa=1,2$ and 3 , respectively. For instance, $x_{11}^{\mathrm{i}}$ represents $\mathrm{U}_{\mathrm{Xi}}, \mathfrak{x}_{23}^{\mathrm{i}}$ represents $\Phi_{\mathrm{ri}}$ and $\mathrm{b}_{12}$ represents $\mathrm{F}_{\omega}$, and so forth. To accesses matrices in the blocks of Eq.(25), a superscript $\kappa$ is added to $\mathfrak{A}$ so that $\mathfrak{U}_{\mathrm{ij}}^{K}$ depicts matrices in the block $\mathfrak{U}_{\mathrm{ij}}$ relating to the space, frequency and resistor when $\kappa$ equals to respectively, 1, 2 and 3. The expanded system of Eq.(22) is

$$
\mathfrak{A} \sum_{\mathrm{i}=1}^{\mathrm{R}} \mathfrak{x}^{\mathrm{i}} \approx \mathfrak{b}
$$

The PGD method suggests calculating each $\mathfrak{x}^{\mathrm{i}}$ successively. Namely, in the $\mathrm{m}^{\text {th }}$ enrichment for $\mathrm{m}=1, \cdots, \mathrm{R}, \mathfrak{x}^{\mathrm{m}}$ is calculated on the basis of sum of all its $m-1$ preceding terms. Components $\mathfrak{x}_{\lambda \kappa}^{\mathrm{m}}$ in $\mathfrak{x}^{\mathrm{m}}$ are calculated individually through e.g. fixed point iterations.

\section{B. PGD interpreted as an optimization problem}

Firstly, define suitable subspaces $\delta_{\lambda \kappa}$ so that $\mathfrak{x}_{\lambda \kappa}^{\mathrm{m}} \in \delta_{\lambda \kappa}$. Examples of such subspaces are $\delta_{11} \subset \mathbb{C}^{\mathrm{N}_{\mathrm{u}} \times 1}$ and $\mathcal{S}_{23} \subset$ $\mathbb{C}^{\mathrm{N}_{\mathrm{r}} \times 1}$. As a result, $\mathfrak{x}_{\lambda}^{\mathrm{m}}$ belongs $\mathcal{S}_{\lambda}:=\mathcal{S}_{\lambda 1} \times \mathcal{S}_{\lambda 2} \times \mathcal{S}_{\lambda 3}$ and $\mathfrak{x}^{\mathrm{m}} \in \mathcal{S}:=\left\{\left\{\begin{array}{ll}z_{1} & z_{2}\end{array}\right\}^{\mathrm{T}} ; z_{\lambda} \in \mathcal{S}_{\lambda}\right.$ for $\left.\lambda=1,2\right\}$. Denote the residual $\mathfrak{b}_{\lambda}-\mathfrak{A}(\lambda,:)\left(\mathfrak{x}^{\{\mathrm{m}-1\}}+\mathfrak{x}^{\mathrm{m}}\right)$ by $\mathfrak{r}_{\lambda}^{\mathrm{m}}$ where the "colon notation" in MATLAB is used to designate the $\lambda$ th row of $\mathfrak{A}$ in $\mathfrak{U}(\lambda,:)$. The new mode $\mathfrak{x}^{\mathrm{m}}$ can be calculated from Eq.(30) which is sometimes referred to as the nearest Kronecker product problem [24].

$$
\mathfrak{x}^{\mathrm{m}}=\arg \min _{\mathfrak{x}^{\mathrm{m}^{*}}}\left\|\mathrm{r}_{\lambda}^{\mathrm{m}}\left(\mathfrak{x}^{\mathrm{m} *}\right)\right\|_{2} \text { for } \lambda=1,2
$$

Or find $\mathfrak{x}^{\mathrm{m}} \in \mathcal{S}$ such that for $\lambda=1,2$

$$
\left(\mathfrak{U}(\lambda,:) \mathfrak{x}^{\mathrm{m}}, \mathfrak{x}_{\lambda}^{\mathrm{m}^{\prime}}\right)_{\mathcal{S}_{\lambda}}=\left(\mathfrak{r}_{\lambda}^{\mathrm{m}-1}, \mathfrak{x}_{\lambda}^{\mathrm{m}^{\prime}}\right)_{\mathcal{S}_{\lambda}} \forall \mathfrak{x}_{\lambda}^{\mathrm{m}^{\prime}} \in \mathcal{S}_{\lambda}
$$

where $(\star, \star)_{\delta_{\lambda}}$ is the inner product on $\delta_{\lambda}$ and it is calculated as $(\star, \star)_{\delta_{\lambda}}:=\prod_{\kappa=1}^{3}\left(\star_{\kappa},{ }{ }_{\kappa}\right)_{\delta_{\lambda \kappa}}$ [23]. Since the vector $\star_{\kappa}$ is complex valued, the inner product $\left(\star{ },{ }^{\star}{ }_{K}\right)_{\delta_{\lambda \kappa}}$ is $\star_{K}{ }^{H} \cdot \star_{K}$ in which $\mathrm{H}$ denotes the conjugate transpose. When $\mathfrak{U}$ is Hermitian, the above equation can be reformulated as

$$
\mathfrak{x}_{\lambda}^{\mathrm{m}^{\prime}}{ }^{\mathrm{H}} \mathfrak{A}(\lambda,:) \mathfrak{x}^{\mathrm{m}}=\mathfrak{x}_{\lambda}^{\mathrm{m}^{\prime \prime}}{ }_{\mathfrak{r}_{\lambda}^{\mathrm{m}-1}}^{\mathrm{m}}
$$

Unfortunately, in our case the operator is symmetric but nonHermitian because matrices in Eq.(25) are symmetric and real-valued, terms involving multiplication of this matrix with the imaginary unit make $\mathfrak{A}$ non-Hermitian. Consequent- ly, Eq.(30) needs to be calculated using, e.g. the least square method. This is achieved by replacing $\mathfrak{A}$ in Eq.(32) by $\widetilde{\mathfrak{A}}$, being $\widetilde{\mathfrak{A}}:=\mathfrak{A}^{\mathrm{T}} \mathfrak{A}$. However, it is not recommended because first, $\widetilde{\mathfrak{A}}$ becomes dense rather than sparse as $\mathfrak{A}$ is. Second, convergence rate of the resultant PGD system is significantly slowed down [10]. Nevertheless, as reported in [12], the approach based on Eq.(32) proves efficient to capture good approximations in many cases even $\mathfrak{A}$ is non-Hermitian. We consider Eq.(32) in the following.

\section{Implementing details}

Solving Eq.(32) is a nonlinear problem as products of unknowns are involved. With the ADM, it is assumed that the two components other than the $\mu^{\text {th }}$ in the $\mathrm{m}^{\text {th }}$ mode are known when calculating for $\mathfrak{x}_{\lambda \mu}^{\mathrm{m}}$, as in Eq.(33). Assumed known variables need to be initialized where for the first step they can be arbitrary (or simply taken as ones) and for the subsequent, solutions of the former enrichment can be taken.

$$
\mathfrak{x}_{\lambda}^{\mathrm{m}^{\prime}}=\mathfrak{x}_{\lambda \mu}^{\mathrm{m}}{ }^{\prime} \bigotimes_{\kappa \neq \mu} \mathfrak{x}_{\lambda \kappa}^{\mathrm{m}}
$$

Substituting Eq.(33) into Eq.(32) and cancelling $\mathfrak{x}_{\lambda \mu}^{\mathrm{m}}{ }^{\prime}$ on each side yields a linear system for $\mathfrak{x}_{\lambda \mu}^{\mathrm{m}}$

$$
\left(\bigotimes_{\kappa \neq \mu} \mathfrak{x}_{\lambda \kappa}^{\mathrm{m}}\right)^{\mathrm{H}} \mathfrak{A}(\lambda,:) \mathfrak{x}^{\mathrm{m}}=\left(\bigotimes_{\kappa \neq \mu} \mathfrak{x}_{\lambda \kappa}^{\mathrm{m}}\right)^{\mathrm{H}} \mathfrak{r}_{\lambda}^{\mathrm{m}-1}
$$

Recall that vectors associated with $\mathrm{X}, \omega$ and $\mathrm{r}$ are calculated when $\mu$ takes value from 1 to 3 while mechanical displacements and electrical potentials are calculated when $\lambda$ takes 1 and 2 . This process enforces orthogonality between the residual $\mathfrak{r}_{\lambda}^{\mathrm{m}}$ and the subspace $\delta_{\lambda}$. Iterations are involved using the ADM. Denote the iteration counter as p and the value of $\mathfrak{x}_{\lambda \mu}^{\mathrm{m}}$ calculated at the $\mathrm{p}^{\text {th }}$ iteration as $\mathfrak{x}_{\lambda \mu}^{\mathrm{m}, \mathrm{p}}$. Iterations are terminated when either a maximum number $n_{\text {In }}$ for $p$ is attained or the following convergence criterion is met

$$
\max _{\lambda} \prod_{\mu=1}^{3} \frac{\left\|\mathfrak{x}_{\lambda \mu}^{\mathrm{m}, \mathrm{p}}-\mathfrak{x}_{\lambda \mu}^{\mathrm{m}, \mathrm{p}-1}\right\|_{\mathrm{F}}}{\left\|\mathfrak{x}_{\lambda \mu}^{\mathrm{m}, \mathrm{p}-1}\right\|_{\mathrm{F}}}<\varepsilon_{\text {In }}
$$

where $\|\star\|_{\mathrm{F}}$ stands for the Frobenius norm and $\varepsilon_{\text {In }}$ is a predefined criterion. It is noted that calculating $\mathfrak{x}_{\lambda}^{\mathrm{m}, \mathrm{p}}$ as $\mathfrak{x}_{\lambda 1}^{\mathrm{m}, \mathrm{p}} \otimes$ $x_{\lambda 2}^{\mathrm{m}, \mathrm{p}} \otimes \mathfrak{x}_{\lambda 3}^{\mathrm{m}, \mathrm{p}}$ and then comparing the difference between $x_{\lambda}^{\mathrm{m}, \mathrm{p}}$ and $x_{\lambda}^{\mathrm{m}, \mathrm{p}-1}$ is less practical as it is memory intensive and reduces the speed. Indeed, the relation $\left\|\mathfrak{x}_{\lambda}^{\mathrm{m}, \mathrm{p}}\right\|_{\mathrm{F}}=$ $\prod_{\mu=1}^{3}\left\|\mathfrak{X}_{\lambda \mu}^{\mathrm{m}, \mathrm{p}}\right\|_{\mathrm{F}}$ indicates that it has the same effect as what described above.

The enriched vectors associating with the space and frequency are normalized before they are added into the new mode. Actually, one can normalize any two in the three vectors. The purpose is to prevent cases where one vector tends 
to infinity while another tends to zero, which causes numerical overflow. Normally, both $\epsilon_{\text {In }}$ and $n_{p}$ are set to ensure that iterations do not take too long.

The enrichment proceeds till $\mathrm{m}>R$, the maximum enrichment number or the following criterion is met

$$
\max _{\lambda} \frac{\left\|x_{\lambda}^{\mathrm{m}}\right\|_{\mathrm{F}}}{\left\|\mathfrak{x}_{\lambda}^{1}\right\|_{\mathrm{F}}}<\varepsilon_{\text {out }}
$$

which means the last mode is small enough compared with the first mode. Eq.(36) is rudimentary whereas it is efficient. Deciding the values for $\varepsilon_{\text {out }}$ is problem dependent.

Once the enrichment process is finished, an approximation $\mathfrak{x}^{\text {pgd }}$ with the R rank-one tensors $\left\{\begin{array}{lllll}\mathfrak{x}_{\lambda}^{1} & \mid & \cdots & \mid & \mathfrak{x}_{\lambda}^{\mathrm{R}}\end{array}\right\}$ (denoted as $x_{\lambda}^{\{R\}}$ ) to the real solution can be recovered using Eq.(23). To evaluate the accuracy of $\mathfrak{x}^{\text {pgd }}$, its relative error $\varepsilon_{\text {rel }}$ w.r.t. the solution obtained using the conventional approach $\mathfrak{x}^{\text {con }}:=\left\{\mathfrak{x}_{1}^{\text {con }} \mathfrak{x}_{2}^{\text {con }}\right\}^{\mathrm{T}}$ can be calculated.

$$
\varepsilon_{\text {rel }}=\max _{\lambda} \frac{\left\|x_{\lambda}^{\text {pgd }}-x_{\lambda}^{\text {con }}\right\|_{F}}{\left\|x_{\lambda}^{\text {con }}\right\|_{F}}
$$

\section{Updating strategy}

In [10] the above process is referred to as the progress Galerkin PGD. Its convergence can be accelerated via updating the results after each enrichment. The idea is to impose orthogonality between $\mathfrak{r}_{\lambda}^{\mathrm{m}}$ and the subspace $\left(\mathcal{S}_{\lambda}\right)^{\mathrm{m}}:=$ $\times_{\mu=1,2,3}\left(\mathcal{S}_{\lambda \mu}\right)^{\mathrm{m}}$, as expressed in Eq.(38).

$$
\mathfrak{x}_{\lambda}^{1 \mathrm{H}}\left(\mathfrak{b}_{\lambda}-\mathfrak{A}(\lambda,:) \mathfrak{x}^{\{m\}}\right)=0, \quad \mathrm{l}=1,2, \ldots, \mathrm{m}
$$

The ADM can be employed which consists in solving all $\mathrm{m}$ components $x_{\lambda \mu}^{1}$ while fixing the other two set of $m$ components $x_{\lambda \kappa}^{1}$ for $\kappa \neq \mu$ and take $\mu=1,2,3$ in turn. However, it is found in our numerical tests that the convergence rate can be extremely slow, especially for a large m. In other words, increasing iteration numbers has insignificant effects on the improvement of convergence. Hence, only one iteration in the updating is considered here. It also worthies noting that when fixing all frequency and resistor related vectors and updating space related vectors, the size of the system to be solved is the square of $\mathrm{m} \times\left(\mathrm{N}_{\mathrm{u}}+\mathrm{N}_{\varphi}\right)$ that quickly becomes too large to be tractable as $\mathrm{m}$ increases. Therefore, vectors of a large number of dofs (spatial components) are not updated. The implementing is summarized in the algorithm chart.

\section{NUMERICAL EXAMPLES}

\section{A. Model description}

Consider a cantilevered piezoelectric $\mathrm{EH}$ that is fixed onto a base. Acceleration are applied on the base which makes the
EH deform, acting as mechanical excitation for the EH. To model the device, it is more convenient to consider displacements of the EH w.r.t. the base. Thus, we assume that the $\mathrm{EH}$ is fixed on the left while subject to an acceleration in the vertical direction (Fig.4a). Dimensions of the bimorph are adopted from [18] where two PZT-5H layers of $27 \times 3.2 \times 0.258 \mathrm{~mm}^{3}$ and a brass substrate of size $27 \times 3.2 \times 0.115 \mathrm{~mm}^{3}$ are connected in series. Their material properties are summarized in Table I.

Six hundred excitation frequencies equally spaced between 0 and $3 \mathrm{kHz}$ are considered. The acceleration is taken constant $\left(9.81 \mathrm{~m} / \mathrm{s}^{2}\right)$ for all frequencies. To compare simulation results with measurements reported in [18], a set of six resistors are chosen (being $100 \Omega, 1 \mathrm{k} \Omega, 10 \mathrm{k} \Omega, 91 \mathrm{k} \Omega$, $500 \mathrm{k} \Omega$ and $910 \mathrm{k} \Omega$ ). An area representing the air is added surrounding the EH. In the mesh, the air area is set to be relatively larger than the $\mathrm{EH}$ domain. A total of 76824 tetrahedral elements with 14783 vertices are generated (Fig.4b). Not that only half of the domain needs to be calculated due to the vertical symmetric surface in the middle.
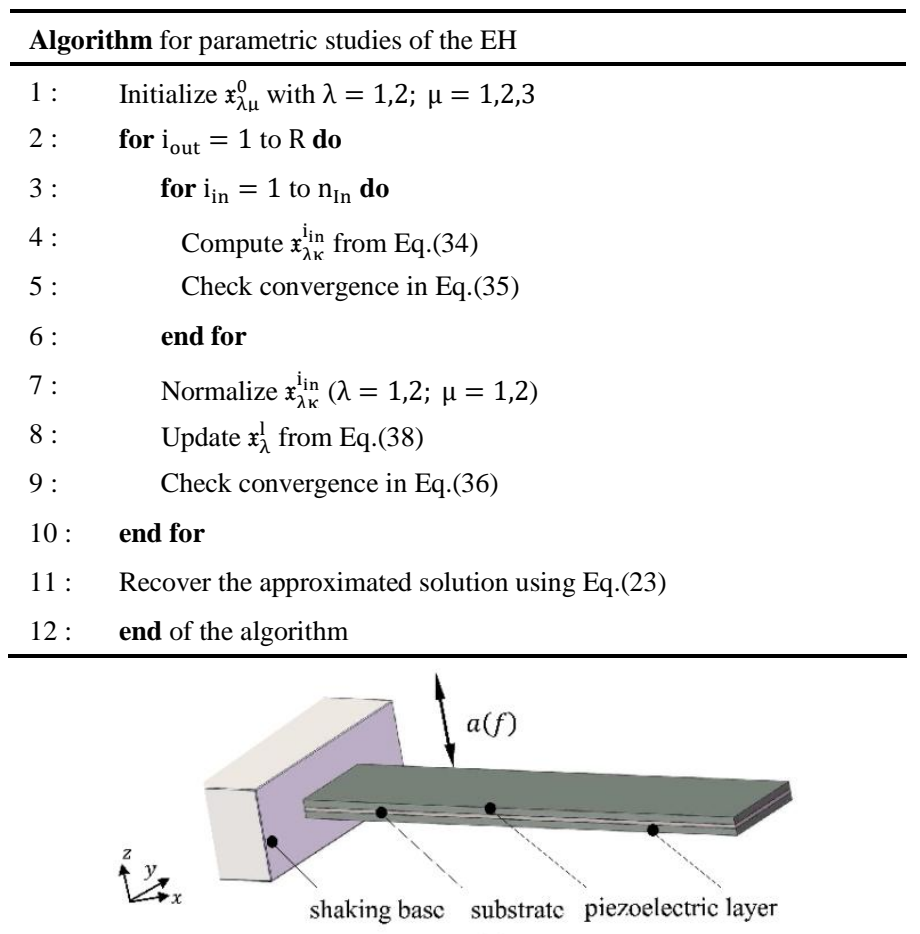

(a)

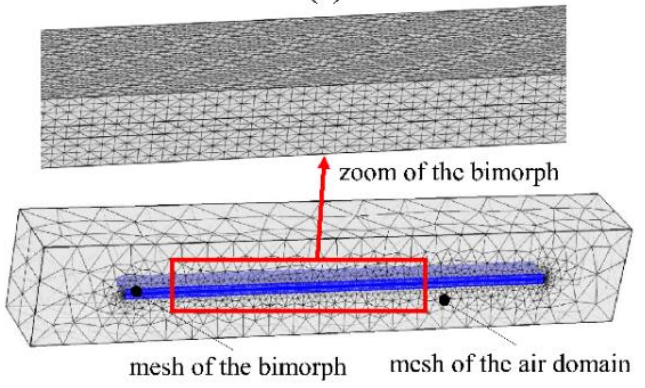

(b)

Fig. 4. Model configuration (a) the cantilevered piezoelectric EH, (b) mesh 


\section{B. Simulation results}

In the first test, parameters for the PGD approach are set so that the fixed point iteration stopping criterion is $10^{-3}$ while that of the enrichment process is $10^{-5}$. Maximum number of iterations and enrichments are set to 10 and 100 , respectively (influence of different criterions are addressed in the next section). The same model is also solved using the conventional approach in which one loop for all samples of the resistor and the other for frequency are included. Both simulations are carried out in MATLAB on a workstation of 32G RAM and an Intel Xeon 3.5 GHz processor.

TABLE I

MATERIAL PROPERTIES Substrate (brass)

\begin{tabular}{lcc}
\hline & \multicolumn{2}{c}{ Substrate (brass) } \\
\hline Mass density & \multicolumn{2}{c}{$9000\left(\mathrm{~kg} \mathrm{~m}^{-3}\right)$} \\
Young's Modulus & \multicolumn{1}{c}{$\begin{array}{c}105 \times 10^{9}\left(\mathrm{~N} \mathrm{~m}^{-2}\right) \\
\text { Poisson's ratio }\end{array}$} & 0.23 \\
\hline Piezoelectric (PZT-5H) & \\
\hline Mass density & & $7500\left(\mathrm{~kg} \mathrm{~m}^{-3}\right)$ \\
Elastic constants & $c_{11}=12.72$ & $c_{12}=8.02$ \\
& $c_{13}=8.47$ & $c_{33}=11.74$ \\
Piezoelectric constants & $c_{44}=2.30$ & $\left(\times 10^{10} \mathrm{~N} \mathrm{~m}^{-2}\right)$ \\
& $e_{31}=-6.62$ & $e_{33}=23.24$ \\
Relative permittivity & $e_{15}=17.03$ & $\left(\mathrm{Cm}^{-2}\right)$ \\
\hline
\end{tabular}

TABLE II

CPU TIME, SOLUTION SIZE AND RESULTS OF DIFFERENT APPROACHES

\begin{tabular}{|c|c|c|c|}
\hline & $\begin{array}{c}\text { CPU time } \\
\text { (s) }\end{array}$ & $\begin{array}{l}\text { Solutions } \\
\text { size }(\mathrm{Mb})\end{array}$ & $\begin{array}{c}\text { Relative } \\
\text { error }\end{array}$ \\
\hline Conventional & 11228 & \multirow{2}{*}{1144} & \multirow{2}{*}{ reference } \\
\hline P-Conventional & 5125 & & \\
\hline PGD & 3094 & \multirow{2}{*}{30.72} & 0.178 \\
\hline U-PGD & 2483 & & $8.01 * 10^{-6}$ \\
\hline
\end{tabular}

The total consumed CPU time, memory required to store the solutions for both approaches and relative error between them are presented in Table II. The conventional approach is carried out both in serial and parallel (the latter is referred to as P-Conventional where the parfor function in MATLAB is used). For the PGD approach, both PGD with and without updating are tested. The former is referred to as U-PGD. PGD and U-PGD undergoes the same amount of enrichments and have solutions of the same size. Solutions of the conventional approaches are taken as the reference for evaluating accuracy of the PGD solutions, based on Eq.(37).

As can be observed, parallel implementation of the conventional approach shortens the calculating time to less than half of the serial counterpart, meanwhile CPUs are fully charged throughout, unlike in other cases where CPUs are only slightly charged. On the other hand, both PGD simulations take less time than P-Conventional in which U-PGD consumes less than half CPU time as the P-Conventional does (that is less than one quarter of the serial conventional version). Differences in CPU time between the PGD and conventional approaches can be explained from their algorithm complexities. Define $\mathcal{O}_{\mathrm{S}}$ as the complexity to solve the FE system Eq.(22) for one combination of $\omega$ and $r$. Thus, complexity of the conventional approach is $\mathrm{N}_{\omega} \times \mathrm{N}_{\mathrm{r}} \times \mathcal{O}_{\mathrm{S}}$. For the PGD approach, the most time-consuming part is calculating the space associated components since $\mathrm{N}_{\omega}$ and $\mathrm{N}_{\mathrm{r}}$ are much smaller than $\mathrm{N}_{\mathrm{u}}$ and $\mathrm{N}_{\varphi}$. Its complexity can be estimated as $\sum_{\mathrm{i}=1}^{\mathrm{R}} \tilde{\mathrm{n}}_{\mathrm{in}}^{\mathrm{i}} \times \mathcal{O}_{\mathrm{S}}$ where $\tilde{\mathrm{n}}_{\mathrm{in}}^{\mathrm{i}}$ is the number of iterations in the ith enrichment. Therefore, the ratio of complexities of the two approaches is $\mathrm{N}_{\omega} \times \mathrm{N}_{\mathrm{r}} / \sum_{\mathrm{i}=1}^{\mathrm{R}} \tilde{\mathrm{n}}_{\mathrm{in}}^{\mathrm{i}}$. Larger this ratio is more time shall be saved with the proposed approach. Another advantage with the PGD approach is that only space of orders of magnitude less is demanded to store the simulation results (in our case the ratio of required space for the conventional and PGD approach is $\left(\mathrm{N}_{\mathrm{u}}+\mathrm{N}_{\varphi}\right) \times \mathrm{N}_{\omega} \times \mathrm{N}_{\mathrm{r}}$ versus $\left(\mathrm{N}_{\mathrm{u}}+\mathrm{N}_{\varphi}+2 \mathrm{~N}_{\omega}+2 \mathrm{~N}_{\mathrm{r}}\right) \times R$ ). It is also observed that the updating plays an important role in accelerating convergence rate of the PGD (see Fig.5) which leads to more accurate solutions.

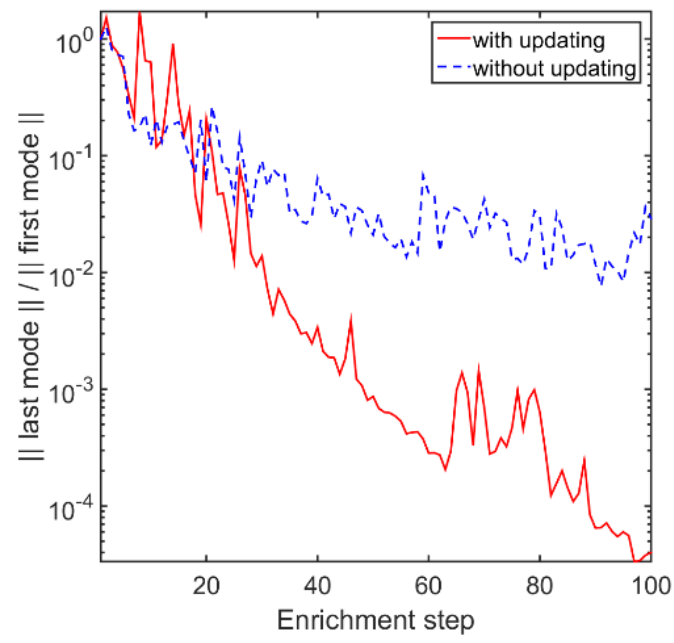

Fig. 5. Convergence of enrichment for with and without updating

In Fig.6 and Fig.7, space and frequency components for the first eight modes are depicted, respectively. As can be seen, only bending modes (none torsion nor other high order modal shapes) appear in the space components which is due to the fact that frequencies chosen in our example only cover the first two eigen frequencies of the bimorph. For the frequency components, most peak values locate near the eigen frequencies of the system. In Fig. 8 are presented impedance components for $R=91 k \Omega$ whose counterparts for other values show a similar trend. 

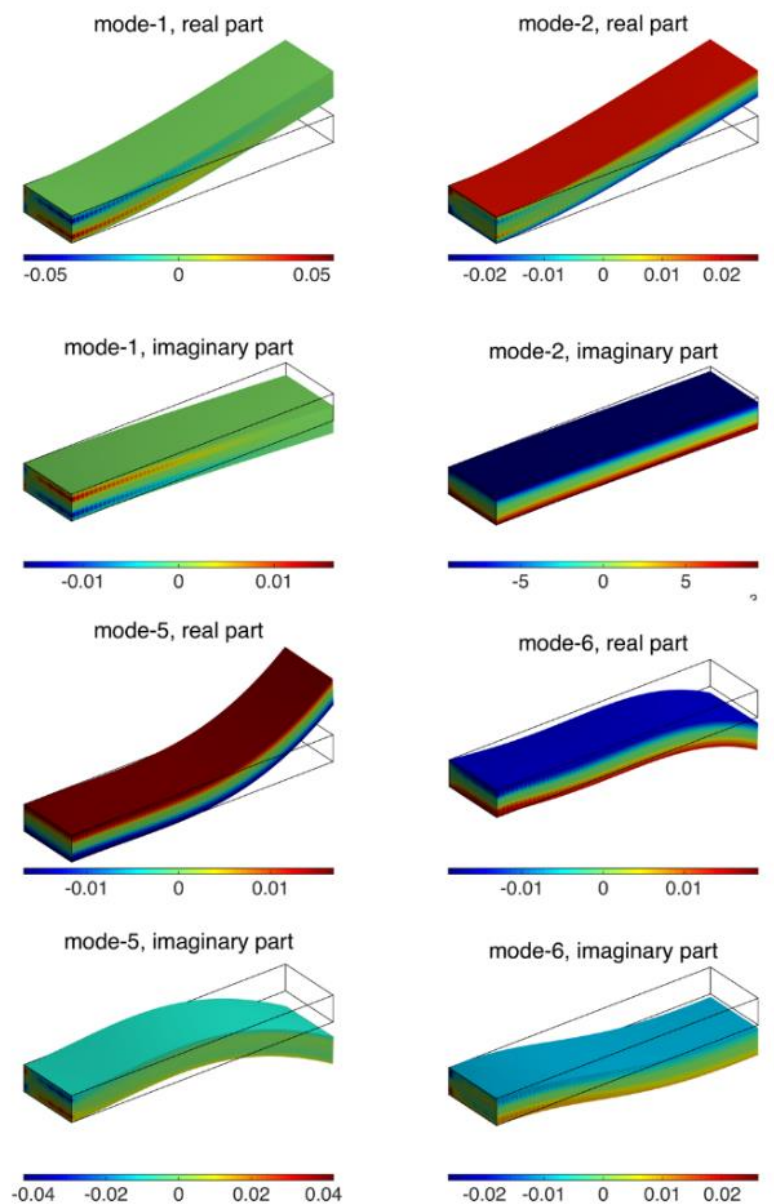

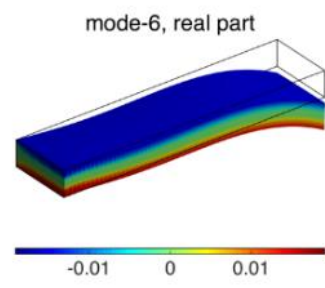

mode-6, imaginary part

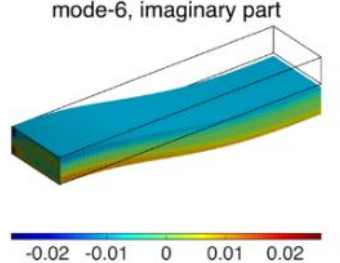

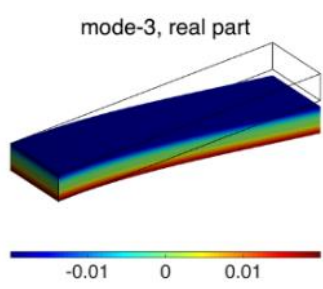
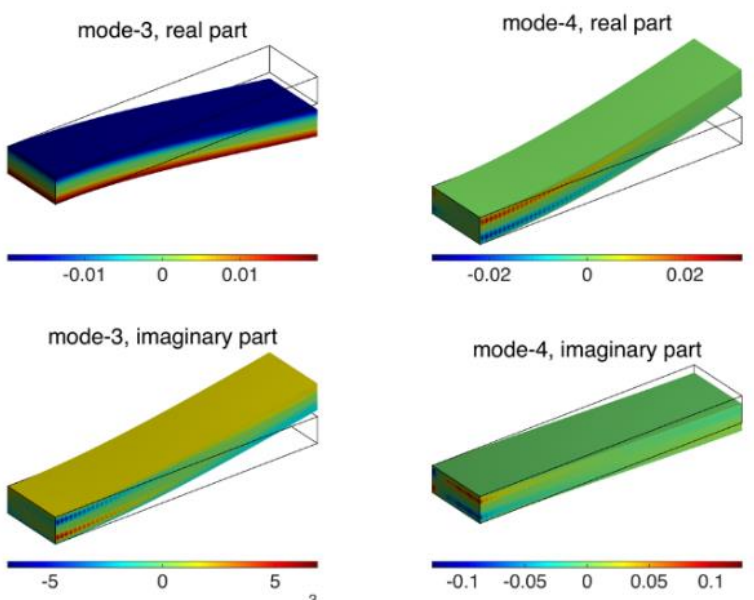

mode-7, real part
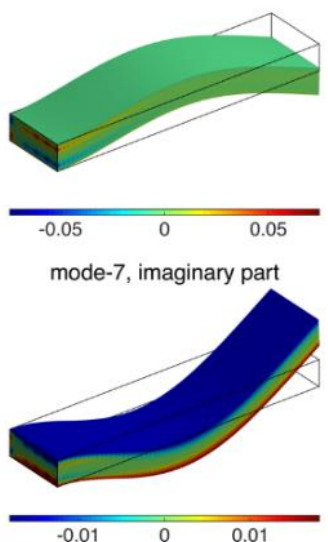
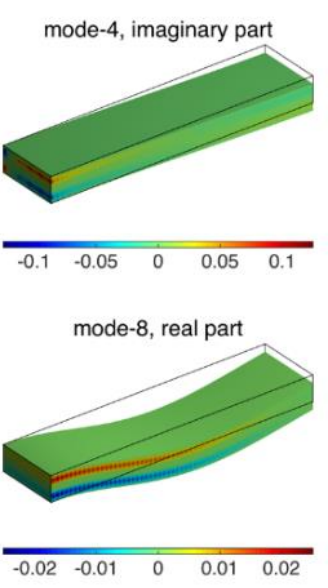

mode-8, imaginary part

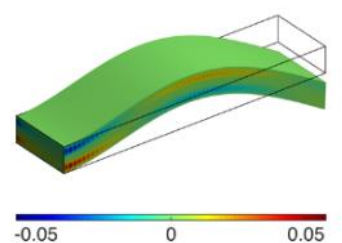

Fig. 6. Space components of the 1 st to the 8 th enrichment (displacements are depicted by the deformation from the original positions while potentials are represented by the color; the area for air is not shown)
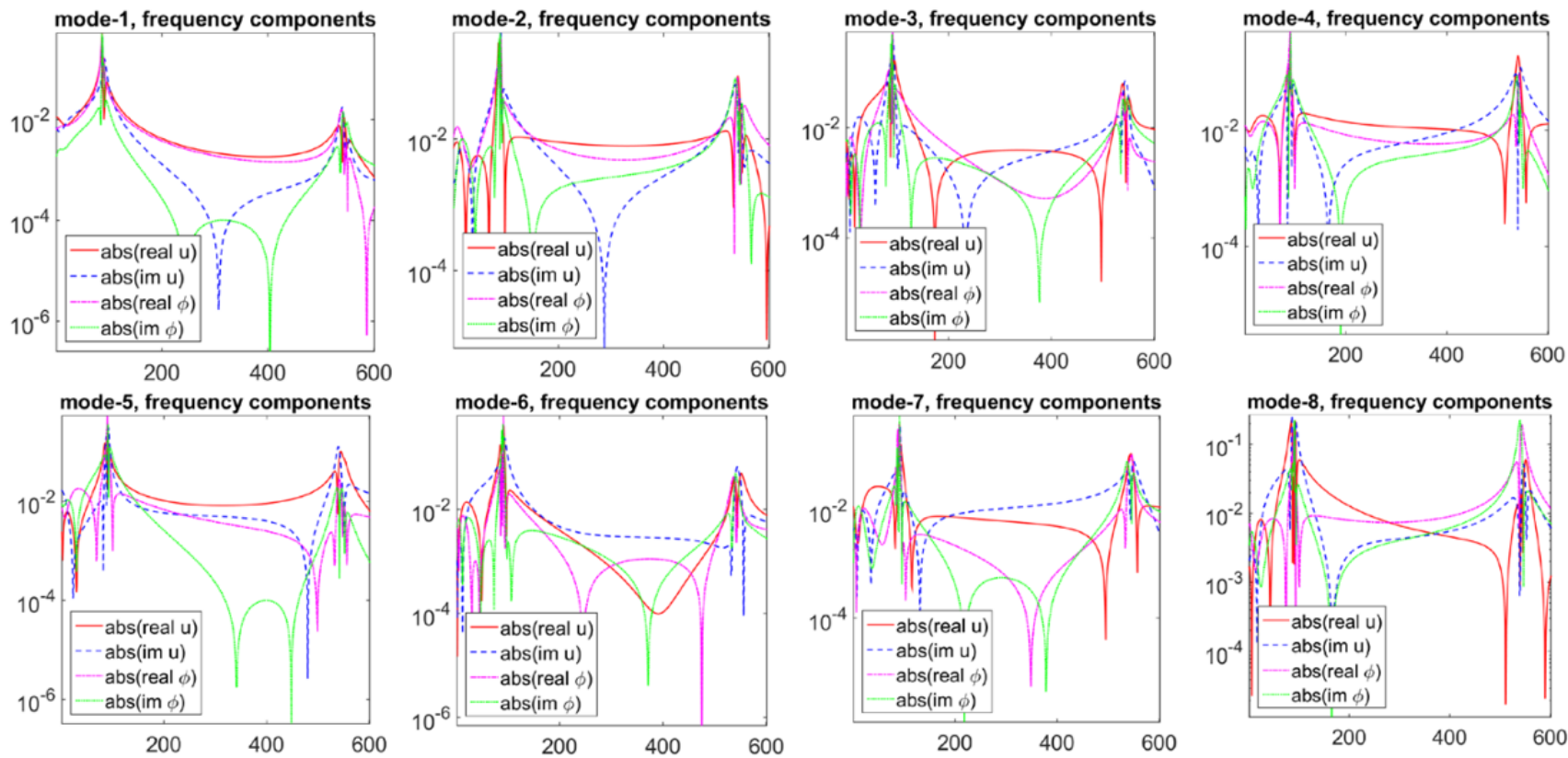

Fig. 7. Frequency components of the 1 st to the 8 th enrichment

For the validation of our results obtained with 100 enrichments, voltages between electrode 1 and 4 are extracted from the PGD results. This voltage under different frequencies and resistors are shown in Fig.9a. It is found that our simulated results compare favorably with the experimental results in the literature (c.f. Fig.4a in [18]). In Fig.9b, the harvested power, or power consumed on the resistors are depicted. As can be seen, the resistor of $91 \mathrm{k} \Omega$ is optimal. 


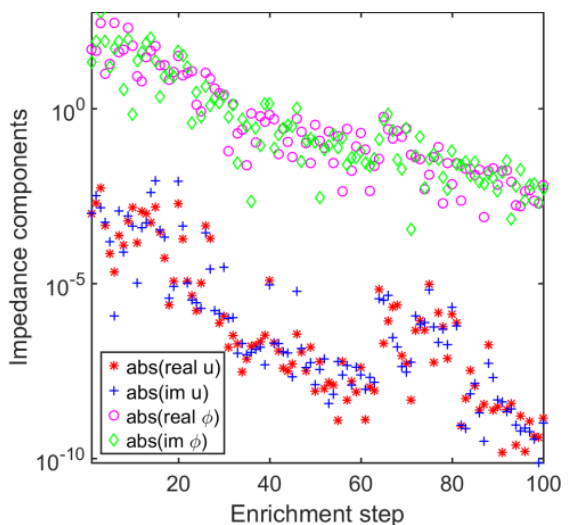

Fig. 8. Impedance components for $\mathrm{R}=91 \mathrm{k} \Omega$

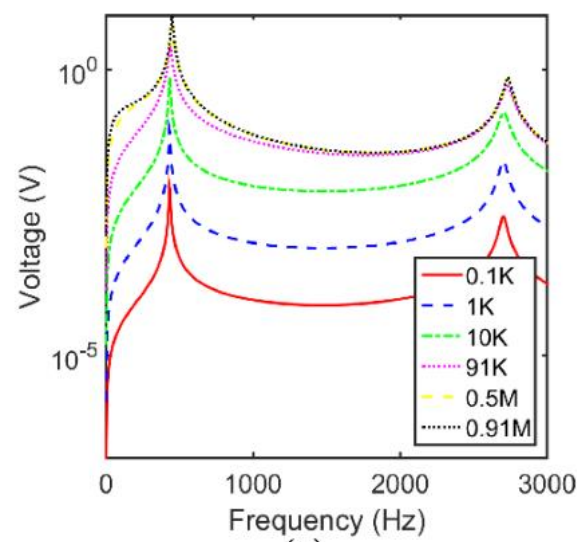

(a)

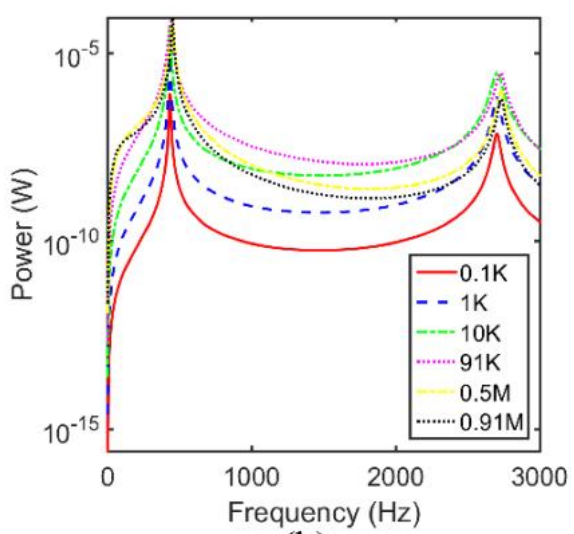

(b)

Fig. 9. Output of the energy harvester (a) generated voltage, (b) power consumed by the electrical load

TABLE III

STUDY ON PARAMETERS OF THE INNER LOOPS $\left(\mathrm{N}_{\mathrm{OUT}}=100, \mathrm{E}_{\text {OUT }}=10^{-5}\right)$

\begin{tabular}{|c|c|c|c|c|c|c|c|c|c|c|c|c|}
\hline \multirow[b]{2}{*}{$\varepsilon_{\mathrm{IN}}$} & \multicolumn{3}{|c|}{$n_{\mathrm{IN}}=5$} & \multicolumn{3}{|c|}{$n_{\mathrm{IN}}=10$} & \multicolumn{3}{|c|}{$n_{\mathrm{IN}}=20$} & \multicolumn{3}{|c|}{$n_{\mathrm{IN}}=30$} \\
\hline & $n_{\text {ite }}$ & $\begin{array}{l}\varepsilon_{\text {conv }} \\
\times 10^{-5}\end{array}$ & $\begin{array}{c}\varepsilon_{\text {rel }} \\
\times 10^{-5}\end{array}$ & $n_{\text {ite }}$ & $\begin{array}{l}\varepsilon_{\mathrm{conv}} \\
\times 10^{-5}\end{array}$ & $\begin{array}{c}\varepsilon_{\text {rel }} \\
\times 10^{-5}\end{array}$ & $n_{\text {ite }}$ & $\begin{array}{l}\varepsilon_{\text {conv }} \\
\times 10^{-5}\end{array}$ & $\begin{array}{c}\varepsilon_{\text {rel }} \\
\times 10^{-5}\end{array}$ & $n_{\text {ite }}$ & $\begin{array}{c}\varepsilon_{\mathrm{conv}} \\
\times 10^{-5}\end{array}$ & $\begin{array}{c}\varepsilon_{\text {rel }} \\
\times 10^{-5}\end{array}$ \\
\hline $10^{-2}$ & 480 & 2.2 & 7.2 & 624 & 2.0 & 6.8 & 608 & 0.82 & 5.6 & 609 & 0.97 & 5.6 \\
\hline $10^{-3}$ & 495 & 3.7 & 7.8 & 839 & 3.9 & 8.0 & 798 & 0.88 & 5.8 & 999 & 0.98 & 5.8 \\
\hline $10^{-4}$ & 498 & 2.0 & 6.7 & 937 & 2.2 & 6.5 & 1052 & 0.95 & 6.1 & 1575 & 0.99 & 6.0 \\
\hline $10^{-5}$ & 500 & 52 & 44 & 863 & 0.96 & 7.7 & 1119 & 0.97 & 5.6 & 1706 & 0.88 & 5.6 \\
\hline
\end{tabular}

TABLE IV

STUDY ON PARAMETERS OF THE OUTER LOOPS $\left(\mathrm{N}_{\mathrm{IN}}=10, \mathrm{E}_{\mathrm{IN}}=10^{-3}\right)$

\begin{tabular}{|c|c|c|c|c|c|c|c|c|c|c|c|c|}
\hline \multirow[b]{2}{*}{$\varepsilon_{\text {OUT }}$} & \multicolumn{3}{|c|}{$n_{\mathrm{OUT}}=30$} & \multicolumn{3}{|c|}{$n_{\text {OUT }}=50$} & \multicolumn{3}{|c|}{$n_{\text {OUT }}=100$} & \multicolumn{3}{|c|}{$n_{\mathrm{OUT}}=200$} \\
\hline & $n_{\text {ite }}$ & $\begin{array}{c}\varepsilon_{\mathrm{conv}} \\
\times 10^{-2}\end{array}$ & $\begin{array}{c}\varepsilon_{\text {rel }} \\
\times 10^{-2}\end{array}$ & $n_{\text {ite }}$ & $\begin{array}{c}\varepsilon_{\text {conv }} \\
\times 10^{-3}\end{array}$ & $\begin{array}{c}\varepsilon_{\text {rel }} \\
\times 10^{-3}\end{array}$ & $n_{\text {ite }}$ & $\begin{array}{c}\varepsilon_{\text {conv }} \\
\times 10^{-4}\end{array}$ & $\begin{array}{c}\varepsilon_{\text {rel }} \\
\times 10^{-4}\end{array}$ & $n_{\text {ite }}$ & $\begin{array}{c}\varepsilon_{\text {conv }} \\
\times 10^{-5}\end{array}$ & $\begin{array}{c}\varepsilon_{\text {rel }} \\
\times 10^{-4}\end{array}$ \\
\hline $10^{-2}$ & 228 & 0.72 & 4.8 & 228 & 7.2 & 48 & 228 & 72 & 480 & 228 & 720 & 480 \\
\hline $10^{-3}$ & 254 & 5.7 & 5.4 & 417 & 2.7 & 1.9 & 425 & 8.2 & 18 & 425 & 82 & 18 \\
\hline $10^{-4}$ & 254 & 5.7 & 5.4 & 417 & 2.7 & 1.9 & 623 & 0.92 & 3.6 & 623 & 9.2 & 3.6 \\
\hline $10^{-5}$ & 254 & 5.7 & 5.4 & 417 & 2.7 & 1.9 & 835 & 0.17 & 0.69 & 948 & 0.82 & 0.58 \\
\hline
\end{tabular}

\section{Influence of the algorithm parameters}

Additional simulations (U-PGD version) are carried out in order to investigate the influence of different parameters in the novel approach. Results are grouped and presented in tables, one for the maximum loop number and stopping criterion of the fixed point iterations (referred to as inner loops) and the other for enrichments (referred to as outer loops). Results are compared in terms of three items: the total iteration number $\mathrm{n}_{\mathrm{ite}}$, the convergence indicator in the last enrichment $\varepsilon_{\text {conv }}$ calculated with Eq.(36), and the relative error $\varepsilon_{\text {rel }}$ obtained with Eq.(37). It is to be noted that only the order of magnitudes are of concern, because the convergence is not monotone and the values may locally oscillate.
In Table III, the maximum enrichment number is fixed at 100 and the convergence criterion is set to $10^{-5}$. It is found that increasing the maximum iteration number $n_{\text {IN }}$ does not significantly improve the accuracy while the complexity increases dramatically. It is recommended to take $\mathrm{n}_{\mathrm{IN}}$ as small as possible e.g. 5 or 10 whenever possible. However, it is more tricky with $\varepsilon_{\mathrm{IN}}$. When it is too large, solutions in step 4 of the algorithm can be far from the exact ones for which more enrichments are required to keep the approximation precision. However, if it is too small the results can be deteriorated, because more iterations are needed for small $\varepsilon_{\text {IN }}$ 's and it can diverge after certain iterations. Consequently, the 
solved modes are not necessarily more accurate albeit the larger number of iterations.

In Table IV, the maximum iteration number is 10 and the iteration convergence criterion is $10^{-3}$ for all cases. Influences of the outer loop parameters are more evident. As suggested in Eq.(23), a larger $R$ normally leads to a better approximation to the exact solution. Therefore, both smaller $\varepsilon_{\text {OUT }}$ and larger $n_{\text {OUT }}$ can hopefully lead to better approximations. However, it is important to realize that the limit of the approximation precision is decided by various factors, such as parameters of both inner and outer loops, and the updating strategy. As a result, the results are no longer improved to some limit by increasing the number of modes. In our case, a maximum number of 50 at $10^{-5}$ is found to be appropriate.

\section{SUMMARY AND CONCLUSIONS}

A PGD based approach for the parametric study of EH numerical models is proposed in this paper. Compared with conventional approaches, the novel approach is capable to obtain numerical results of almost the same quality while at a much reduced complexity. Using the proposed approach, CPU time for solving parametric problems is effectively reduced along with less required space to store the results. In addition, the proposed approach can be readily implemented. Nevertheless, there is still much space for improvements of the proposed method such as effective methods to solve high dimensional problems in updating and more rigorous error estimators for convergence check. Additionally, solving the three dimensional spatial components dominates the simulation process which can be reduced by PGD based decomposition of the full spatial fields into lower dimensional ones [25] though extra efforts than in a pure structural problem are needed due to the piezoelectric coupling. Such problems offer tantalizing prospects for future extensions of this work.

\section{REFERENCES}

[1] G. Rozza, D.B.P. Huynh, and A.T.Patera, "Reduced basis approximation and a posteriori error estimation for affinely parametrized elliptic coercive partial differential equations", Arch. Comput. Method Eng., vol. 15 , no. 3 , pp. 229-275, 2008

[2] F.Chinesta, and P.Ladeveze, "A short review on model order reduction based on proper generalized decomposition", Arch. Comput. Method Eng., vol. 18, no. 4, pp. 395-404, 2011

[3] F. Chinesta, R. Keunings, and A. Leygue, The proper generalized decomposition for advanced numerical simulations: a primer, Springer International Publishing, 2013

[4] A.Nouy, "A low-rank methods for high-dimensional approximation and model order reduction", arXiv: 1511.01554 [math.NA], 2015

[5] L.Grasedyck, D.Kressner, and C.Tobler, "A literature survey of lowrank tensor approximation techniques", GAMM-Mitteilugen vol. 36, no. 1 , pp. $53-78,2013$
[6] E.Pruliere, F.Chinesta, and A.Ammar, "On the deterministic solution of multidimensional parametric models using the proper generalized decomposition”, Math. Comput. Simulat., vol. 81, no. 4, pp. 791-810, 2010

[7] S.Nirromandi, D.Gonzales, I.Alfaro, et al, "Real-time simulation of biological soft tissues : a PGD approach", Int. J. Numer. Method Biomed. Eng., vol. 29, no. 5, pp. 586-600, 2013.

[8] J.V.Aguado, "Advanced strategies for the separated formulation of problems in the proper generalized decomposition framework", Ph.D. dissertation, SPIGA, Université Nantes Anger Le Mans, Nante, France, 2015

[9] D.Modesto, S.Zlotnik, and A.Huerta, "Proper generalized decomposition for parameterized Helmholtz problems in heterogeneous and unbounded domains: Application to harbor agitation", Comput. Methods Appl. Mech. Eng., vol. 295, pp. 127-149, 2015

[10] A.Nouy, "A priori model reduction through proper generalized decomposition for solving time-dependent partial differential equations", Comput. Methods Appl. Mech. Eng., vol. 199, no. 23, pp. 1603-1626, 2010

[11] M.Billaud-Friess, A.Nouy, and O.Zahm, "A tensor approximation method based on ideal minimal residual formulations for the solution of high-dimensional problems", ESAIM Math. Model. Numer. Anal., vol. 48, pp. 1777-1806, 2014

[12] M.Chevreuil and A.Nouy, "A model order reduction based on proper generalized decomposition for the propagation of uncertainty in structural dynamics”, Int. J. Numer. Meth. Eng., vol. 89, no. 2, pp. 241-68, 2012

[13] P.Ladevèze, Nonlinear computational structural mechanics: new approaches and non-incremental methods of calculation, Springer International Publishing, 2012

[14] T.Henneron and S.Clénet, "Proper generalized decomposition method applied to solve 3D magnetoquasi-static field problems coupling with external electric circuits", IEEE Trans. Magn., vol. 51, no. 6, pp. 7208910, Jun. 2015

[15] Z.Qin, H.Talleb and Z.X.Ren, “A proper generalized decomposition based solver for nonlinear magneto-thermal problems", IEEE Trans. Magn., vol. 52, no. 2, pp. 7400209, Feb. 2016

[16] H.Talleb and Z.X.Ren, "Finite element modeling of a magnetoelectric laminate composites in considering nonlinear and load effects for energy harvesting", J. Alloys Compd., vol. 615, no. 5, pp. 64-74, 2014

[17] H.Talleb and Z.X.Ren, "Finite element modeling of a magnetoelectric energy transducer including the load effect", IEEE Trans. Magn., vol. 51, no. 6, pp. 7401305, Mar. 2015

[18] S.Zhao and A.Erturk, "Elecroelastic modeling and experimental validation of piezoelectric energy harvesting from broadband random vibrations of cantilevered bimorphs", Smart Mater. Struct., vol. 22, no. 1, pp. 015002,2013

[19] N.G.Elvin, and A.A.Elvin, "A coupled finite element -circuit simulation model for analyzing piezoelectric energy generators", J. Intel. Mat. Sys. Str., vol. 20, no. 5, pp. 587-595, 2009

[20] IEEE Standard on Piezoelectricity, ANSI/IEEE Standard. 176-1987, 1987

[21] Z.Qin, H.Talleb, J.Y.Duquesne, et al, "Finite element modeling of thermos-elastic attenuation in piezoelectric surface acoustic wave devices”, IEEE Trans. Magn., vol. 51, no. 3, pp. 7401903, Mar. 2015

[22] J.S.Wang, and D.F. Ostergaard, "A finite element circuit coupled simulation method for piezoelectric transducer", IEEE Int. Ultrason. Symp., vol. 2, pp. 1105-1108, Oct. 1999

[23] T.G.Kolda and B.W.Bader, "Tensor decomposition and applications", SIAM Rev., vol. 51, no. 3, pp. 455-500, 2009

[24] G.Golub, and C. van Loan, Matrix computations, 4th ed. Johns Hopkins Press, 2013

[25] P.Vidal, L.Gallimard, O.Polit, "Proper generalized decomposition and layer-wise approach for the modeling of composite plate structures", Int. J. Solids Struct., vol. 50, no.14-15, pp.2239-2250, July 2013 